Antarctic Science 26(6), 674-686 (2014) @ Antarctic Science Ltd 2014. This is an Open Access article, distributed under the terms of the Creative Commons Attribution licence (http://creativecommons.org/licenses/by/3.0/), which permits unrestricted re-use,

distribution, and reproduction in any medium, provided the original work is properly cited.

doi:10.1017/S0954102014000613

\title{
Drivers of abrupt Holocene shifts in West Antarctic ice stream direction determined from combined ice sheet modelling and geologic signatures
}

\author{
C.J. FOGWILL ${ }^{1}$, C.S.M. TURNEY ${ }^{1}$, N.R. GOLLEDGE ${ }^{2,3}$, D.H. ROOD ${ }^{4}$, K. HIPPE ${ }^{5,6}$, L. WACKER ${ }^{6}$, R. WIELER ${ }^{5}$, \\ E.B. RAINSLEY ${ }^{7}$ and R.S. JONES ${ }^{2}$ \\ ${ }^{1}$ Climate Change Research Centre, University of New South Wales, Sydney, NSW 2052, Australia \\ ${ }^{2}$ Antarctic Research Centre, Victoria University of Wellington, Wellington 6140, New Zealand \\ ${ }^{3}$ GNS Science, Avalon, Lower Hutt 5011, New Zealand \\ ${ }^{4}$ Scottish Universities Environmental Research Centre (SUERC), East Kilbride G75 0QF, UK \\ ${ }^{5}$ Institute of Geochemistry and Petrology, ETH Zürich, CH-8092 Zürich, Switzerland \\ ${ }^{6}$ Institute for Particle Physics, ETH Zürich, CH-8093 Zürich, Switzerland \\ ${ }^{7}$ Unaffiliated \\ c.fogwill@unsw.edu.au
}

\begin{abstract}
Determining the millennial-scale behaviour of marine-based sectors of the West Antarctic Ice Sheet (WAIS) is critical to improve predictions of the future contribution of Antarctica to sea level rise. Here highresolution ice sheet modelling was combined with new terrestrial geological constraints (in situ ${ }^{14} \mathrm{C}$ and ${ }^{10} \mathrm{Be}$ analysis) to reconstruct the evolution of two major ice streams entering the Weddell Sea over 20000 years. The results demonstrate how marked differences in ice flux at the marine margin of the expanded Antarctic ice sheet led to a major reorganization of ice streams in the Weddell Sea during the last deglaciation, resulting in the eastward migration of the Institute Ice Stream, triggering a significant regional change in ice sheet mass balance during the early to mid Holocene. The findings highlight how spatial variability in ice flow can cause marked changes in the pattern, flux and flow direction of ice streams on millennial timescales in this marine ice sheet setting. Given that this sector of the WAIS is assumed to be sensitive to ocean-forced instability and may be influenced by predicted twenty-first century ocean warming, our ability to model and predict abrupt and extensive ice stream diversions is key to a realistic assessment of future ice sheet sensitivity.
\end{abstract}

Received 13 May 2014, Accepted 14 July 2014

Key words: cosmogenic isotopes, ice stream dynamics, in situ ${ }^{14} \mathrm{C}$, marine ice sheet instability, Weddell Sea, West Antarctic Ice Sheet

\section{Introduction}

Recent observations of rapidly accelerating West Antarctic outlet glaciers have prompted a radical shift in the way the sensitivity of marine-terminating ice sheets to ocean forcing is viewed (Rignot et al. 2014). Critical to this debate is the influence of subglacial topography on marine-based ice sheet dynamics (commonly referred to as the marine ice sheet instability hypothesis) where positive ice-loss feedbacks may occur when the grounding line is both below sea level and within a basin which deepens towards the centre of the ice sheet (Weertman 1974, Thomas \& Bentley 1978). Decadal-scale changes consistent with this mechanism have been implicated in several key outlets of the West Antarctic Ice Sheet (WAIS) (Rignot et al. 2014), suggesting that even small changes at the margins of the Antarctic ice sheets may trigger far-reaching changes in the interior of the Antarctic ice sheet through ice streams, narrow corridors of enhanced ice flow, which control the mass balance of the
Antarctic ice sheets (Cuffey 2011, Golledge et al. 2012, Rignot et al. 2014). However, it remains unclear whether such a long-term transformation in ice sheet dynamics will take place, potentially leading to future collapse and associated rapid sea level rise (Bamber et al. 2009, Rignot et al. 2014). Therefore, understanding the mechanisms that control enhanced flow is key to predicting future WAIS stability (Cuffey 2011, Rignot et al. 2011).

The Weddell Sea embayment (WSE) of Antarctica potentially offers significant insights into this debate. Today the extensive Filchner-Ronne Ice Shelf of the Weddell Sea is partially sustained by the inflow of nine large ice streams that together drain $22 \%$ of Antarctica, yet its detailed history of deglaciation since the Last Glacial Maximum (LGM), and particularly during the Holocene, remains poorly constrained (Larter et al. 2012, Stolldorf et al. 2012, Siegert et al. 2013, Hillenbrand et al. 2014). Whilst contemporary satellite remote sensing suggests a modest elevation of the ice sheet surface across much of the region in recent decades (Rignot et al. 2011), there is 
a mounting body of evidence that indicates ice stream drainage patterns in the region were markedly different during the Holocene, implying that the region is sensitive to external forcings and thus may be vulnerable to past and potentially future change (Siegert et al. 2013). Evidence for this comes from interpretations of airborne radar-echo sounding (RES), marine geophysical investigations and satellite imagery, which suggests there has been substantial late Holocene reconfiguration of the ice streams in the Weddell Sea; however, the timing and, critically, the mechanisms driving these changes remain uncertain (Siegert et al. 2013). Understanding these mechanisms is critical given twenty-first century projections of ocean warming in the region (Hellmer et al. 2012, Fogwill et al. 2014), and the presence of extensive subglacial basins upstream of the present-day grounding line (Ross et al. 2012).

To assess the response of ice stream configuration in the Weddell Sea to external forcing since the LGM this study generates new high-resolution, whole-continent ice sheet model simulations for comparison with detailed terrestrial and marine geochronological constraints. The combination of these detailed geological ice sheet constraints with the high-resolution palaeo ice sheet model simulations to examine the drivers of ice sheet change over the last 20000 years allows the response of ice streams to ocean forcing and sea level rise during the transition between the glacial and interglacial world to be investigated, improving our understanding of ice dynamic responses of the ice streams in the Weddell Sea and wider WAIS to ocean perturbations. Recent studies (e.g. Larter et al. 2012) postulate that three major cross-shelf troughs may have played a role in controlling WSE dynamics during deglaciation, the Rutford Trough (or Ronne Depression), the Hughes Trough and the Thiel Trough (or Filchner or Crary Trough). The combination of high-resolution ice sheet modelling and geological constraints in this study allows the role of these features to be explored more fully.

\section{Ice sheet model simulations}

Here the results of high-resolution ice sheet modelling experiments that investigate the dynamic response of an LGM-configuration Antarctic ice sheet to ocean forcing (Golledge et al. 2012, 2013) are presented. The parallel ice sheet model (PISM) is used, a 3-D, thermomechanical, continental ice sheet model, constrained by published geological data that define lateral and vertical extents of the expanded Antarctic ice sheets around the time of the LGM (Golledge et al. 2012). The model combines the shallow-ice and shallow-shelf approximation equations across the entire domain. Therefore, the model is able to capture the dynamic behaviour within grounded ice

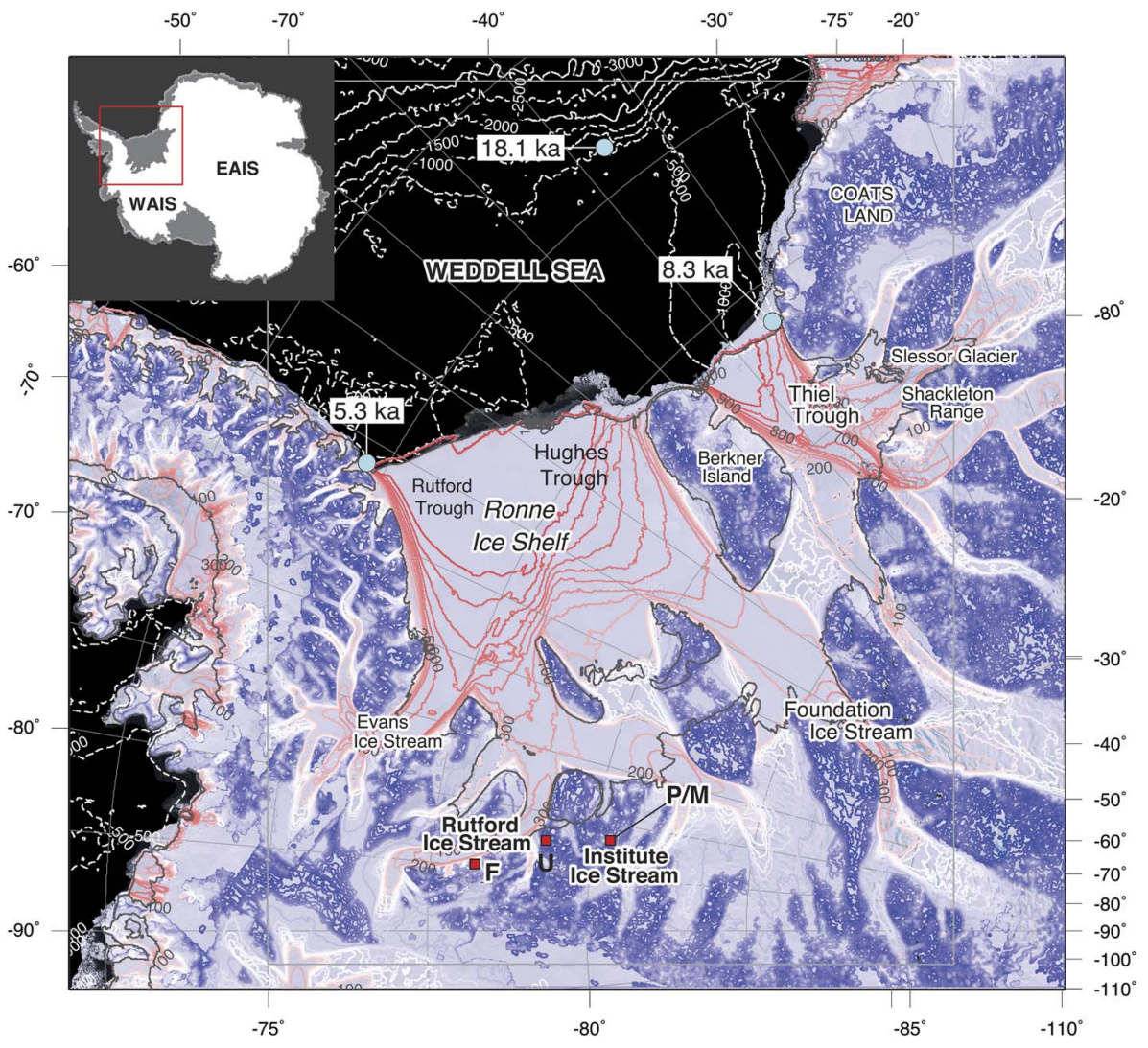

Fig. 1. Weddell Sea embayment (WSE) indicating the sampling locations next to the Rutford and Institute ice streams. Ice sheet surface velocity data (Rignot et al. 2011) highlight the locations of the major ice streams in light colours, and ice rises and slow moving regions in the WSE in darker blue. The sites of marine cores and associated minimum ages for grounding line retreat based upon marine radiocarbon ages (Hillenbrand et al. 2014) are also shown. F = Flower Hills, $\mathrm{U}=$ Union Glacier, $\mathrm{P} / \mathrm{M}=$ Patriot and Marble hills. 
of Antarctic ice sheets and simulate the drawdown of interior ice by ice streams at high resolution $(5 \mathrm{~km})$. In this study, the model uses proxy-based interpretations of oceanic (Lisiecki et al. 2005, Imbrie et al. 2006) and atmospheric (Petit et al. 1999) changes during the last glacial cycle and employs boundary distributions from modified Bedmap topography (Le Brocq et al. 2010), temperature and precipitation fields from gridded datasets (Comiso 2000, Van de Berg 2006), and a spatially varying geothermal heat flux interpolation (Shapiro \& Ritzwoller 2004).

The ice sheet model computes ice thickness and temperature changes, isostatic depression of topography, migration of grounding lines and the growth of ice shelves. Interaction between modelled ice shelves and their surrounding ocean is accounted for using a mass balance determination based on heat flux across the ice-water boundary. Our perturbation experiments use isochronous changes to oceanic heat flux and sea level values. The ice sheet model simulations are based on ocean-perturbation experiments in which the oceanic heat flux and sea level are isochronously increased from $30 \%$ to $100 \%$ of glacial to interglacial transition values, and by $25 \mathrm{~m}$ and $50 \mathrm{~m}$, with respect to LGM values, representative of Holocene values. The response of the ice sheet is considered in terms of changes in velocity and ice thickness which together yield mass flux and, importantly, changes in ice flow direction.

\section{Geological and geochronological constraints}

To provide a temporal context for the modelled ice stream response, new and recalibrated existing geochronological data are used to reconstruct the geometric and temporal changes in the ice streams feeding the western part of the Weddell Sea following the LGM. The terrestrial record constrains altitudinal changes of the former ice stream surface, whereas offshore marine records, from radiocarbon dating of glaciomarine sediments overlying the subglacial deposits, constrain the lateral extent of the ice sheet in the WSE. Both are required to reconstruct the 3 -D changes in ice stream geometry and investigate palaeo ice volume changes.

\section{Marine geochronological constraints}

The limited available analyses of marine sediment cores (including radiocarbon) from the outer and inner continental shelf of the WSE are used to constrain the lateral extent of the ice sheet, and provide the timing of ice sheet grounding line retreat and establishment of open water conditions (Larter et al. 2012, Stolldorf et al. 2012,
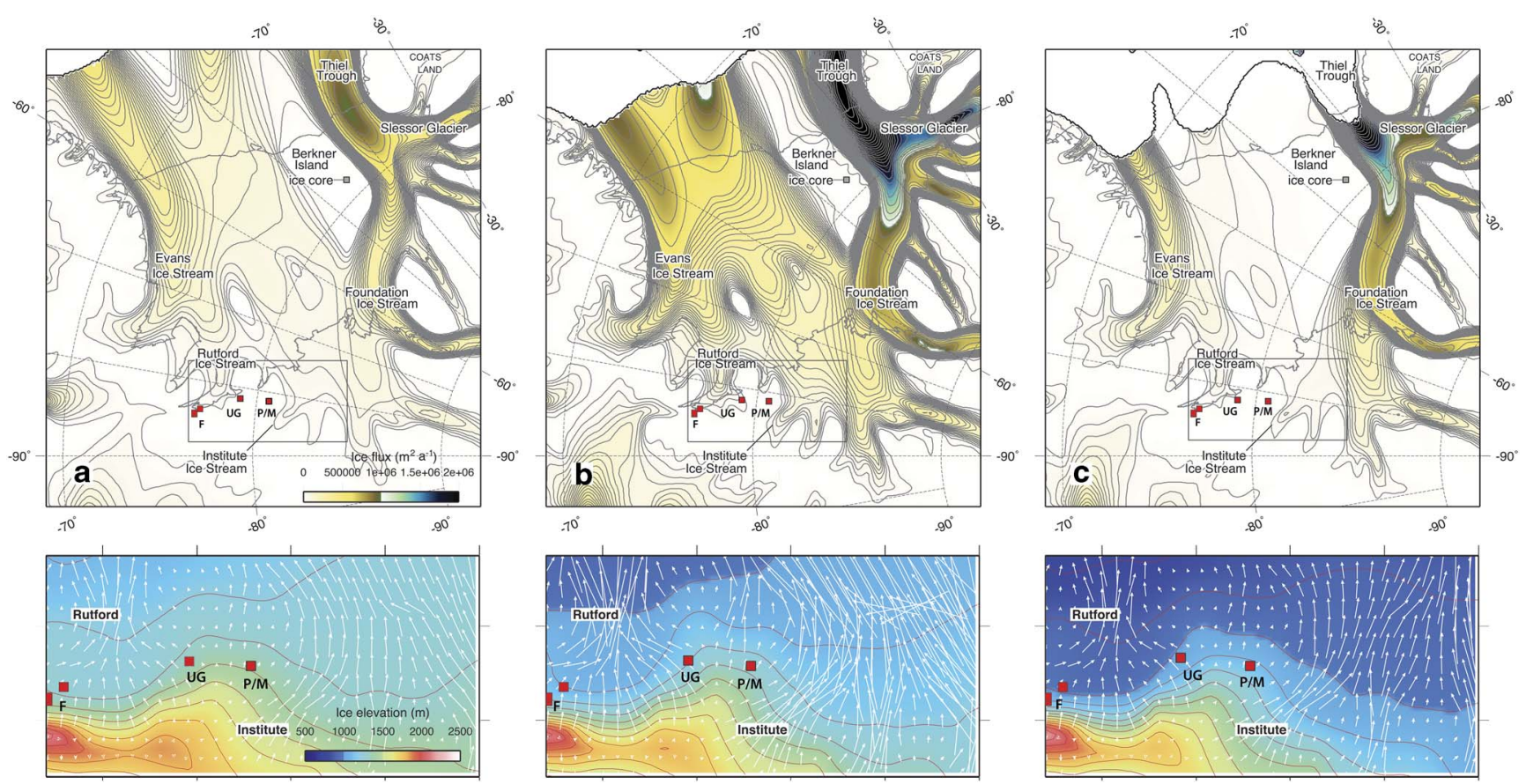

Fig. 2. Simulated regional ice flux (upper panels), together with ice flow direction (white arrows) and ice sheet surface elevation of the Rutford and Institute ice streams (lower panel). a. Post-LGM conditions. b. Initial response to imposed ocean forcing leads to widespread acceleration of ice flow at principal outlets at $c .15000$ model years. c. Continued ice recession then leading to capture of the Institute Ice Stream by the Thiel Trough outlet during the late to mid Holocene. Ice flow vectors in the area of interest illustrate the change in flow direction taking place between time slices and red squares show the sample locations. $\mathrm{F}=\mathrm{Flower}$ Hills, $\mathrm{UG}=$ Union Glacier, $\mathrm{P} / \mathrm{M}=$ Patriot and Marble hills. 
Table I. ${ }^{10} \mathrm{Be}$ cosmogenic isotope data from the Patriot and Marble hills recording changes in the Institute Ice Stream, and data from the Flower Hills and Union Glacier recording changes in the Rutford Ice Stream (Fogwill et al. 2012).

\begin{tabular}{|c|c|c|c|c|c|c|c|c|c|c|c|c|c|c|c|c|c|c|c|}
\hline Sample name & $\begin{array}{l}\text { Latitude } \\
\left({ }^{\circ} \mathrm{S}\right)\end{array}$ & $\begin{array}{l}\text { Longitude } \\
\left({ }^{\circ} \mathrm{W}\right)\end{array}$ & $\begin{array}{l}\text { Elevation } \\
\text { (m) }\end{array}$ & $\begin{array}{l}\text { Elevation/ } \\
\text { pressure }\end{array}$ & $\begin{array}{l}\text { Thickness } \\
(\mathrm{cm})\end{array}$ & Density & Shielding ${ }^{\mathrm{a}}$ & $\begin{array}{l}\text { Erosion } \\
\text { rate }\end{array}$ & ${ }^{10} \mathrm{Be}\left(\right.$ at g $\left.{ }^{-1}\right)$ & $\begin{array}{c} \pm{ }^{10} \mathrm{Be} \\
\left(\text { at g }^{-1}\right)^{\mathrm{b}}\end{array}$ & ${ }^{10} \mathrm{Be}$ standard & ${ }^{26} \mathrm{Al}\left(\mathrm{at} \mathrm{g}^{-1}\right)$ & $\pm{ }^{26} \mathrm{Al}\left(\mathrm{at} \mathrm{g}^{-1}\right)$ & ${ }^{26} \mathrm{Al}$ standard & $\begin{array}{l}{ }^{10} \text { Be exposure } \\
\text { age (years) } \\
\text { P }_{\text {Global }}{ }^{c}\end{array}$ & $\begin{array}{l} \pm \text { External } \\
\text { uncertainty } \\
\text { (years) }\end{array}$ & $\begin{array}{c}{ }^{10} \mathrm{Be} \\
\text { exposure age } \\
\text { (years) } \mathrm{P}_{\mathrm{NZ}}\end{array}$ & $\begin{array}{l} \pm \text { External } \\
\text { uncertainty } \\
\text { (years) }\end{array}$ & $\begin{array}{c}\text { Extraction /AMS } \\
\text { analysis } \\
\text { undertaken at }^{\mathrm{d}}\end{array}$ \\
\hline \multicolumn{20}{|l|}{ Patriot Hills } \\
\hline CF-01-08 & -80 & -81 & 760 & ant & 5 & 2.65 & 0.98 & 0 & $1.62 \mathrm{E}+05$ & $8.24 \mathrm{E}+03$ & NIST_27900 & $0.00 \mathrm{E}+00$ & $0.00 \mathrm{E}+00$ & KNSTD & 14273 & 1442 & 16642 & 924 & ED-SUERC \\
\hline CF-02-08 & -80 & -81 & 760 & ant & 5 & 2.65 & 0.99 & 0 & $9.11 \mathrm{E}+03$ & $1.29 \mathrm{E}+03$ & NIST_27900 & $0.00 \mathrm{E}+00$ & $0.00 \mathrm{E}+00$ & KNSTD & 792 & 132 & 923 & 132 & ED-SUERC \\
\hline CF-03-08 & -80 & -81 & 762 & ant & 5 & 2.65 & 0.98 & 0 & $5.99 \mathrm{E}+03$ & $4.00 \mathrm{E}+02$ & NIST_27900 & $0.00 \mathrm{E}+00$ & $0.00 \mathrm{E}+00$ & KNSTD & 525 & 58 & 612 & 43 & ED-SUERC \\
\hline CF-08-08 & -80 & -81 & 936 & ant & 5 & 2.65 & 0.98 & 0 & $5.47 \mathrm{E}+06$ & $5.29 \mathrm{E}+04$ & NIST_27900 & $0.00 \mathrm{E}+00$ & $0.00 \mathrm{E}+00$ & KNSTD & 460394 & 45242 & 548064 & 15009 & ED-SUERC \\
\hline CF-09-08 & -80 & -81 & 1004 & ant & 5 & 2.65 & 0.99 & 0 & $5.65 \mathrm{E}+06$ & $6.96 \mathrm{E}+04$ & NIST_27900 & $0.00 \mathrm{E}+00$ & $0.00 \mathrm{E}+00$ & KNSTD & 442082 & 43401 & 525873 & 15036 & ED-SUERC \\
\hline CF-13-08 & -80 & -81 & 989 & ant & 5 & 2.65 & 0.97 & 0 & $1.06 \mathrm{E}+06$ & $1.75 E+04$ & NIST_27900 & $0.00 \mathrm{E}+00$ & $0.00 \mathrm{E}+00$ & KNSTD & 78420 & 7071 & 91722 & 2563 & ED-SUERC \\
\hline CF-14-08 & -80 & -81 & 935 & ant & 5 & 2.65 & 0.97 & 0 & $4.61 \mathrm{E}+05$ & $1.25 \mathrm{E}+04$ & NIST_27900 & $0.00 \mathrm{E}+00$ & $0.00 \mathrm{E}+00$ & KNSTD & 35352 & 3245 & 41269 & 1449 & ED-SUERC \\
\hline CF-17-08 & -80 & -81 & 826 & ant & 5 & 2.65 & 0.97 & 0 & $5.77 \mathrm{E}+05$ & $1.54 \mathrm{E}+04$ & NIST_27900 & $0.00 \mathrm{E}+00$ & $0.00 \mathrm{E}+00$ & KNSTD & 48841 & 4493 & 57036 & 1992 & ED-SUERC \\
\hline CF-19-08 & -80 & -81 & 816 & ant & 5 & 2.65 & 0.97 & 0 & $2.66 \mathrm{E}+05$ & $6.10 \mathrm{E}+03$ & NIST_27900 & $0.00 \mathrm{E}+00$ & $0.00 \mathrm{E}+00$ & KNSTD & 22568 & 2039 & 26325 & 838 & ED-SUERC \\
\hline CF-21-08 & -80 & -81 & 774 & ant & 5 & 2.65 & 0.99 & 0 & $4.86 \mathrm{E}+04$ & $1.25 \mathrm{E}+03$ & NIST_27900 & $0.00 \mathrm{E}+00$ & $0.00 \mathrm{E}+00$ & KNSTD & 4176 & 379 & 4868 & 164 & ED-SUERC \\
\hline CF-24-08 & -80 & -81 & 761 & ant & 5 & 2.65 & 0.97 & 0 & $3.64 \mathrm{E}+04$ & $4.84 \mathrm{E}+02$ & NIST_27900 & $0.00 \mathrm{E}+00$ & $0.00 \mathrm{E}+00$ & KNSTD & 3227 & 284 & 3761 & 96 & ED-SUERC \\
\hline CF-25-08 & -80 & -81 & 940 & ant & 5 & 2.65 & 0.97 & 0 & $6.25 \mathrm{E}+05$ & $1.59 \mathrm{E}+04$ & NIST_27900 & $0.00 \mathrm{E}+00$ & $0.00 \mathrm{E}+00$ & KNSTD & 47870 & 4385 & 55912 & 1898 & ED-SUERC \\
\hline CF-28-08 & -80 & -81 & 879 & ant & 5 & 2.65 & 0.97 & 0 & $6.89 \mathrm{E}+05$ & $1.80 \mathrm{E}+04$ & NIST_27900 & $0.00 \mathrm{E}+00$ & $0.00 \mathrm{E}+00$ & KNSTD & 55760 & 5129 & 65143 & 2251 & ED-SUERC \\
\hline CF-29-08 & -80 & -81 & 879 & ant & 5 & 2.65 & 0.97 & 0 & $3.95 \mathrm{E}+05$ & $1.05 \mathrm{E}+04$ & NIST_27900 & $0.00 \mathrm{E}+00$ & $0.00 \mathrm{E}+00$ & KNSTD & 31777 & 2910 & 37086 & 1286 & ED-SUERC \\
\hline CF-31-08 & -80 & -81 & 863 & ant & 5 & 2.65 & 0.97 & 0 & $1.79 \mathrm{E}+05$ & $4.11 \mathrm{E}+03$ & NIST_27900 & $0.00 \mathrm{E}+00$ & $0.00 \mathrm{E}+00$ & KNSTD & 14541 & 1311 & 16958 & 539 & ED-SUERC \\
\hline PAT-01-MJB & -80 & -81 & 1092 & ant & 5 & 2.65 & 0.99 & 0 & $1.34 \mathrm{E}+05$ & $3.79 E+03$ & NIST_27900 & $0.00 \mathrm{E}+00$ & $0.00 \mathrm{E}+00$ & KNSTD & 8748 & 801 & 10204 & 365 & ED-SUERC \\
\hline PAT-03-MJB & -80 & -81 & 1009 & ant & 5 & 2.65 & 0.97 & 0 & $8.70 \mathrm{E}+04$ & $2.65 E+04$ & NIST_27900 & $0.00 \mathrm{E}+00$ & $0.00 \mathrm{E}+00$ & KNSTD & 6214 & 1971 & 7246 & 2217 & ED-SUERC \\
\hline PAT-04-CJF & -80 & -81 & 933 & ant & 5 & 2.65 & 0.97 & 0 & $4.11 \mathrm{E}+05$ & $1.11 \mathrm{E}+04$ & NIST_27900 & $0.00 \mathrm{E}+00$ & $0.00 \mathrm{E}+00$ & KNSTD & 31542 & 2892 & 36816 & 1289 & ED-SUERC \\
\hline PAT-04-MJB & -80 & -81 & 998 & ant & 5 & 2.65 & 0.99 & 0 & $1.39 \mathrm{E}+05$ & $1.00 \mathrm{E}+04$ & NIST_27900 & $0.00 \mathrm{E}+00$ & $0.00 \mathrm{E}+00$ & KNSTD & 9833 & 1112 & 11468 & 864 & ED-SUERC \\
\hline PAT-05-MJB & -80 & -81 & 954 & ant & 5 & 2.65 & 0.97 & 0 & $9.37 \mathrm{E}+04$ & $1.08 \mathrm{E}+04$ & NIST_27900 & $0.00 \mathrm{E}+00$ & $0.00 \mathrm{E}+00$ & KNSTD & 7018 & 1015 & 8184 & 962 & ED-SUERC \\
\hline PAT-08-CJF & -80 & -81 & 1004 & ant & 5 & 2.65 & 0.97 & 0 & $5.36 \mathrm{E}+06$ & $8.50 \mathrm{E}+04$ & NIST_27900 & $0.00 \mathrm{E}+00$ & $0.00 \mathrm{E}+00$ & KNSTD & 426237 & 41944 & 506588 & 15519 & ED-SUERC \\
\hline PAT-10-CJF & -80 & -81 & 1002 & ant & 5 & 2.65 & 0.97 & 0 & $5.90 \mathrm{E}+06$ & $1.05 E+05$ & NIST_27900 & $0.00 \mathrm{E}+00$ & $0.00 \mathrm{E}+00$ & KNSTD & 475596 & 47601 & 566645 & 18410 & ED-SUERC \\
\hline PAT-13-CJF & -80 & -81 & 978 & ant & 5 & 2.65 & 0.97 & 0 & $5.09 \mathrm{E}+06$ & $9.31 E+04$ & NIST_27900 & $0.00 \mathrm{E}+00$ & $0.00 \mathrm{E}+00$ & KNSTD & 412538 & 40667 & 489952 & 15778 & ED-SUERC \\
\hline PAT-14-CJF & -80 & -81 & 968 & ant & 5 & 2.65 & 0.97 & 0 & $1.09 \mathrm{E}+06$ & $2.07 \mathrm{E}+04$ & NIST_27900 & $0.00 \mathrm{E}+00$ & $0.00 \mathrm{E}+00$ & KNSTD & 82187 & 7459 & 96140 & 2844 & ED-SUERC \\
\hline PAT-15-CJF & -80 & -81 & 965 & ant & 5 & 2.65 & 0.97 & 0 & $1.05 E+06$ & $1.42 \mathrm{E}+04$ & NIST_27900 & $0.00 \mathrm{E}+00$ & $0.00 \mathrm{E}+00$ & KNSTD & 79319 & 7113 & 92773 & 2432 & ED-SUERC \\
\hline PAT-16-CJF & -80 & -81 & 960 & ant & 5 & 2.65 & 0.97 & 0 & $7.17 \mathrm{E}+05$ & $1.99 \mathrm{E}+04$ & NIST_27900 & $0.00 \mathrm{E}+00$ & $0.00 \mathrm{E}+00$ & KNSTD & 54058 & 4996 & 63158 & 2263 & ED-SUERC \\
\hline PAT-18-CJF & -80 & -81 & 774 & ant & 5 & 2.65 & 0.97 & 0 & $5.36 \mathrm{E}+06$ & $8.50 \mathrm{E}+04$ & NIST_27900 & $0.00 \mathrm{E}+00$ & $0.00 \mathrm{E}+00$ & KNSTD & 534920 & 54146 & 638944 & 20264 & ED-SUERC \\
\hline PAT-20-CJF & -80 & -81 & 772 & ant & 5 & 2.65 & 0.97 & 0 & $9.52 \mathrm{E}+05$ & $1.03 E+04$ & NIST_27900 & $0.00 \mathrm{E}+00$ & $0.00 \mathrm{E}+00$ & KNSTD & 85311 & 7629 & 99769 & 2485 & ED-SUERC \\
\hline PAT-21-CJF & -80 & -81 & 769 & ant & 5 & 2.65 & 0.97 & 0 & $3.97 \mathrm{E}+05$ & $6.77 \mathrm{E}+03$ & NIST_27900 & $0.00 \mathrm{E}+00$ & $0.00 \mathrm{E}+00$ & KNSTD & 35230 & 3146 & 41113 & 1148 & ED-SUERC \\
\hline PAT-24-CJF & -80 & -81 & 777 & ant & 5 & 2.65 & 0.97 & 0 & $3.77 E+05$ & $7.76 \mathrm{E}+03$ & NIST_27900 & $0.00 \mathrm{E}+00$ & $0.00 \mathrm{E}+00$ & KNSTD & 33201 & 2988 & 38742 & 1172 & ED-SUERC \\
\hline PAT-25-CJF & -80 & -81 & 775 & ant & 5 & 2.65 & 0.97 & 0 & $4.42 \mathrm{E}+05$ & $6.97 \mathrm{E}+03$ & NIST_27900 & $0.00 \mathrm{E}+00$ & $0.00 \mathrm{E}+00$ & KNSTD & 39051 & 3481 & 45580 & 1239 & ED-SUERC \\
\hline PAT-26-CJF & -80 & -81 & 775 & ant & 5 & 2.65 & 0.99 & 0 & $5.43 \mathrm{E}+05$ & $1.16 \mathrm{E}+04$ & NIST_27900 & $0.00 \mathrm{E}+00$ & $0.00 \mathrm{E}+00$ & KNSTD & 47121 & 4265 & 55023 & 1701 & ED-SUERC \\
\hline \multicolumn{20}{|l|}{ Marble Hills } \\
\hline CF-222-08 & -80 & -82 & 1126 & ant & 5 & 2.65 & 0.98 & 0 & $6.21 \mathrm{E}+05$ & $7.61 E+03$ & NIST_27900 & $0.00 \mathrm{E}+00$ & $0.00 \mathrm{E}+00$ & KNSTD & 40106 & 3554 & 46844 & 1183 & ED-SUERC \\
\hline CF-223-08 & -80 & -82 & 1126 & ant & 5 & 2.65 & 0.99 & 0 & $7.06 \mathrm{E}+05$ & $1.86 \mathrm{E}+04$ & NIST_27900 & $0.00 \mathrm{E}+00$ & $0.00 \mathrm{E}+00$ & KNSTD & 45200 & 4149 & 52808 & 1828 & ED-SUERC \\
\hline CF-224-08 & -80 & -82 & 1126 & ant & 5 & 2.65 & 0.99 & 0 & $9.57 \mathrm{E}+05$ & $2.67 \mathrm{E}+04$ & NIST_27900 & $0.00 \mathrm{E}+00$ & $0.00 \mathrm{E}+00$ & KNSTD & 61519 & 5700 & 71923 & 2591 & ED-SUERC \\
\hline CF-225-08 & -80 & -82 & 1032 & ant & 5 & 2.65 & 0.99 & 0 & $8.51 \mathrm{E}+05$ & $2.33 E+04$ & NIST_27900 & $0.00 \mathrm{E}+00$ & $0.00 \mathrm{E}+00$ & KNSTD & 59198 & 5472 & 69192 & 2462 & ED-SUERC \\
\hline CF-227-08 & -80 & -82 & 1032 & ant & 5 & 2.65 & 0.99 & 0 & $5.35 \mathrm{E}+05$ & $1.48 \mathrm{E}+04$ & NIST_27900 & $0.00 \mathrm{E}+00$ & $0.00 \mathrm{E}+00$ & KNSTD & 37011 & 3405 & 43219 & 1538 & ED-SUERC \\
\hline CF-228-08 & -80 & -82 & 986 & ant & 5 & 2.65 & 0.99 & 0 & $8.09 \mathrm{E}+04$ & $1.74 E+03$ & NIST_27900 & $0.00 \mathrm{E}+00$ & $0.00 \mathrm{E}+00$ & KNSTD & 5776 & 518 & 6736 & 206 & ED-SUERC \\
\hline CF-229-08 & -80 & -82 & 986 & ant & 5 & 2.65 & 0.99 & 0 & $6.95 \mathrm{E}+04$ & $1.66 \mathrm{E}+03$ & NIST_27900 & $0.00 \mathrm{E}+00$ & $0.00 \mathrm{E}+00$ & KNSTD & 4961 & 447 & 5785 & 187 & ED-SUERC \\
\hline CF-230-08 & -80 & -82 & 950 & ant & 5 & 2.65 & 0.99 & 0 & $1.28 \mathrm{E}+05$ & $3.06 \mathrm{E}+03$ & NIST_27900 & $0.00 \mathrm{E}+00$ & $0.00 \mathrm{E}+00$ & KNSTD & 9436 & 852 & 11005 & 357 & ED-SUERC \\
\hline CF-231-08 & -80 & -82 & & ant & 5 & 2.65 & 0.99 & 0 & $4.52 \mathrm{E}+03$ & $2.97 \mathrm{E}+02$ & NIST_27900 & $0.00 \mathrm{E}+00$ & $0.00 \mathrm{E}+00$ & KNSTD & & & 388 & 27 & ED-SUERC \\
\hline MAR-02-CJF & -80 & -82 & 1385 & ant & 5 & 2.65 & 0.99 & 0 & $7.27 \mathrm{E}+06$ & $1.76 \mathrm{E}+05$ & NIST_27900 & $0.00 \mathrm{E}+00$ & $0.00 \mathrm{E}+00$ & KNSTD & 411772 & 41226 & 489394 & 18045 & ED-SUERC \\
\hline MAR-04-mjb & -80 & -82 & 1246 & ant & 5 & 2.65 & 0.99 & 0 & $3.61 \mathrm{E}+06$ & $8.94 \mathrm{E}+04$ & NIST_27900 & $2.13 E+07$ & $3.99 \mathrm{E}+05$ & KNSTD & 218357 & 20839 & 257099 & 9043 & ED-SUERC \\
\hline MAR-05-MJB & -80 & -82 & 1192 & ant & 5 & 2.65 & 0.97 & 0 & $1.19 \mathrm{E}+05$ & $2.99 \mathrm{E}+03$ & NIST_27900 & $0.00 \mathrm{E}+00$ & $0.00 \mathrm{E}+00$ & KNSTD & 7287 & 660 & 8501 & 283 & ED-SUERC \\
\hline MAR-06-MJB & -80 & -82 & 1166 & ant & 5 & 2.65 & 0.97 & 0 & $1.27 \mathrm{E}+05$ & $5.72 \mathrm{E}+03$ & NIST_27900 & $5.98 \mathrm{E}+06$ & $1.36 \mathrm{E}+05$ & KNSTD & 7949 & 779 & 9272 & 465 & ED-SUERC \\
\hline MAR-07-cjf & -80 & -82 & 1300 & ant & 5 & 2.65 & 0.99 & 0 & $5.37 \mathrm{E}+05$ & $1.22 \mathrm{E}+04$ & NIST_27900 & $3.17 \mathrm{E}+06$ & $1.46 \mathrm{E}+05$ & KNSTD & 29662 & 2683 & 34640 & 1099 & ED-SUERC \\
\hline MAR-08-CJF & -80 & -82 & 1302 & ant & 5 & 2.65 & 0.99 & 0 & $6.45 \mathrm{E}+05$ & $5.59 \mathrm{E}+04$ & NIST_27900 & $0.00 \mathrm{E}+00$ & $0.00 \mathrm{E}+00$ & KNSTD & 35622 & 4410 & 41612 & 3757 & ED-SUERC \\
\hline MAR-08-MJB & -80 & -82 & 1002 & ant & 5 & 2.65 & 0.97 & 0 & $9.55 \mathrm{E}+04$ & $9.43 E+02$ & NIST_27900 & $0.00 \mathrm{E}+00$ & $0.00 \mathrm{E}+00$ & KNSTD & 6863 & 601 & 8004 & 192 & ED-SUERC \\
\hline MAR-09-cjf & -80 & -82 & 1305 & ant & 5 & 2.65 & 0.99 & 0 & $2.95 \mathrm{E}+05$ & $2.20 \mathrm{E}+03$ & NIST_27900 & $0.00 \mathrm{E}+00$ & $0.00 \mathrm{E}+00$ & KNSTD & 16174 & 1416 & 18878 & 436 & ED-SUERC \\
\hline MAR-10-CJF & -80 & -82 & 1280 & ant & 5 & 2.65 & 0.99 & 0 & $4.65 \mathrm{E}+06$ & $1.04 \mathrm{E}+05$ & NIST_27900 & $0.00 \mathrm{E}+00$ & $0.00 \mathrm{E}+00$ & KNSTD & 277530 & 26701 & 327679 & 11108 & ED-SUERC \\
\hline MAR-10-MJB & -80 & -82 & 974 & ant & 5 & 2.65 & 0.99 & 0 & $1.70 \mathrm{E}+05$ & $3.18 \mathrm{E}+03$ & NIST_27900 & $0.00 \mathrm{E}+00$ & $0.00 \mathrm{E}+00$ & KNSTD & 12284 & 1095 & 14328 & 413 & ED-SUERC \\
\hline MAR-11_cjf & -80 & -82 & 1280 & ant & 5 & 2.65 & 0.99 & 0 & $2.96 \mathrm{E}+05$ & $9.64 \mathrm{E}+03$ & NIST_27900 & $0.00 \mathrm{E}+00$ & $0.00 \mathrm{E}+00$ & KNSTD & 16564 & 1543 & 19334 & 761 & ED-SUERC \\
\hline MAR-11-MJB & -80 & -82 & 810 & ant & 5 & 2.65 & 0.99 & 0 & $2.73 E+04$ & $6.39 \mathrm{E}+02$ & NIST_27900 & $0.00 \mathrm{E}+00$ & $0.00 \mathrm{E}+00$ & KNSTD & 2271 & 204 & 2647 & 85 & ED-SUERC \\
\hline
\end{tabular}


Table I: Continued

\begin{tabular}{|c|c|c|c|c|c|c|c|c|c|c|c|c|c|c|c|c|c|c|c|}
\hline Sample name & $\begin{array}{l}\text { Latitude } \\
\left({ }^{\circ} \mathrm{S}\right)\end{array}$ & $\begin{array}{c}\text { Longitude } \\
\left({ }^{\circ} \mathrm{W}\right)\end{array}$ & $\begin{array}{l}\text { Elevation } \\
\quad(\mathrm{m})\end{array}$ & $\begin{array}{c}\text { Elevation/ } \\
\text { pressure }\end{array}$ & $\begin{array}{l}\text { Thickness } \\
(\mathrm{cm})\end{array}$ & Density & Shielding $^{\mathrm{a}}$ & $\begin{array}{l}\text { Erosion } \\
\text { rate }\end{array}$ & ${ }^{10} \mathrm{Be}\left(\right.$ at $\left.\mathrm{g}^{-1}\right)$ & $\begin{array}{c} \pm^{ \pm 10} \mathrm{Be} \\
\left({ }^{a t} \mathrm{~g}^{-1}\right)^{\mathrm{b}}\end{array}$ & ${ }^{10} \mathrm{Be}$ standard & ${ }^{26} \mathrm{Al}\left(\mathrm{at} \mathrm{g}^{-1}\right)$ & $\pm{ }^{26} \mathrm{Al}\left(\right.$ at g $\left.^{-1}\right)$ & ${ }^{26} \mathrm{Al}$ standard & $\begin{array}{c}{ }^{10} \text { Be exposure } \\
\text { age (years) } \\
\text { P }_{\text {Global }}{ }^{\mathrm{c}}\end{array}$ & $\begin{array}{c} \pm \text { External } \\
\text { uncertainty } \\
\text { (years) }\end{array}$ & $\begin{array}{c}{ }^{10} \mathrm{Be} \\
\text { exposure age } \\
\text { (years) } \mathrm{P}_{\mathrm{NZ}}{ }^{\mathrm{c}}\end{array}$ & $\begin{array}{c} \pm \text { External } \\
\text { uncertainty } \\
\text { (years) }\end{array}$ & $\begin{array}{l}\text { Extraction /AMS } \\
\text { analysis } \\
{\text { undertaken } \text { at }^{\mathrm{d}}}\end{array}$ \\
\hline MAR-12-MJB & -80 & -82 & 807 & ant & 5 & 2.65 & 0.97 & 0 & $3.48 \mathrm{E}+04$ & $2.11 \mathrm{E}+03$ & NIST_27900 & $0.00 \mathrm{E}+00$ & $0.00 \mathrm{E}+00$ & KNSTD & 2962 & 314 & 3452 & 223 & ED-SUERC \\
\hline MAR-13-CJF & -80 & -82 & 1112 & ant & 5 & 2.65 & 0.99 & 0 & $3.38 \mathrm{E}+06$ & $7.60 \mathrm{E}+04$ & NIST_27900 & $0.00 \mathrm{E}+00$ & $0.00 \mathrm{E}+00$ & KNSTD & 229135 & 21782 & 269865 & 9039 & ED-SUERC \\
\hline MAR-16-cjf & -80 & -82 & 1117 & ant & 5 & 2.65 & 0.99 & 0 & $7.66 \mathrm{E}+05$ & $1.83 \mathrm{E}+04$ & NIST_27900 & $4.79 \mathrm{E}+06$ & $1.01 \mathrm{E}+05$ & KNSTD & 49466 & 4512 & 57801 & 1895 & ED-SUERC \\
\hline MAR-17-MJB & -80 & -82 & 959 & ant & 5 & 2.65 & 0.99 & 0 & $1.17 \mathrm{E}+06$ & $2.62 \mathrm{E}+04$ & NIST_27900 & $0.00 \mathrm{E}+00$ & $0.00 \mathrm{E}+00$ & KNSTD & 87256 & 8000 & 102097 & 3270 & ED-SUERC \\
\hline MAR-18-MJB & -80 & -82 & 943 & ant & 5 & 2.65 & 0.99 & 0 & $4.02 \mathrm{E}+05$ & $8.47 \mathrm{E}+03$ & NIST_27900 & $0.00 \mathrm{E}+00$ & $0.00 \mathrm{E}+00$ & KNSTD & 29968 & 2699 & 34979 & 1069 & ED-SUERC \\
\hline MAR-19-CJF & -80 & -82 & 1109 & ant & 5 & 2.65 & 0.99 & 0 & $5.41 \mathrm{E}+05$ & $1.01 \mathrm{E}+04$ & NIST_27900 & $0.00 \mathrm{E}+00$ & $0.00 \mathrm{E}+00$ & KNSTD & 35046 & 3141 & 40926 & 1185 & ED-SUERC \\
\hline MAR-19-MJB & -80 & -82 & 936 & ant & 5 & 2.65 & 0.99 & 0 & $4.07 \mathrm{E}+05$ & $1.26 \mathrm{E}+04$ & NIST_27900 & $0.00 \mathrm{E}+00$ & $0.00 \mathrm{E}+00$ & KNSTD & 30530 & 2837 & 35635 & 1360 & ED-SUERC \\
\hline MAR-20-MJB & -80 & -82 & 900 & ant & 5 & 2.65 & 0.99 & 0 & $3.92 \mathrm{E}+05$ & $7.40 \mathrm{E}+03$ & NIST_27900 & $0.00 \mathrm{E}+00$ & $0.00 \mathrm{E}+00$ & KNSTD & 30339 & 2717 & 35409 & 1029 & ED-SUERC \\
\hline MAR-21-cjf & -80 & -82 & 1040 & ant & 5 & 2.65 & 0.99 & 0 & $3.44 \mathrm{E}+06$ & $8.22 \mathrm{E}+04$ & NIST_27900 & $1.49 \mathrm{E}+07$ & $3.09 \mathrm{E}+05$ & KNSTD & 249066 & 23894 & 293570 & 10218 & ED-SUERC \\
\hline MAR-24-cjf & -80 & -82 & 953 & ant & 5 & 2.65 & 0.99 & 0 & $4.96 \mathrm{E}+05$ & $1.20 \mathrm{E}+04$ & NIST_27900 & $2.90 \mathrm{E}+06$ & $8.03 E+04$ & KNSTD & 36719 & 3341 & 42871 & 1410 & ED-SUERC \\
\hline MAR-24-MJB & -80 & -82 & 1133 & ant & 5 & 2.65 & 0.99 & 0 & $8.12 \mathrm{E}+05$ & $1.53 \mathrm{E}+04$ & NIST_27900 & $0.00 \mathrm{E}+00$ & $0.00 \mathrm{E}+00$ & KNSTD & 51767 & 4661 & 60498 & 1768 & ED-SUERC \\
\hline MAR-26-CJF & -80 & -82 & 879 & ant & 5 & 2.65 & 0.99 & 0 & $8.18 \mathrm{E}+04$ & $4.73 E+03$ & NIST_27900 & $0.00 \mathrm{E}+00$ & $0.00 \mathrm{E}+00$ & KNSTD & 6410 & 670 & 7473 & 463 & ED-SUERC \\
\hline \multicolumn{20}{|l|}{ Flower Hills } \\
\hline FLO-18-CJF & -78 & -85 & 1327 & ant & 5 & 2.65 & 0.985 & 0 & $3.73 E+05$ & $6.78 \mathrm{E}+03$ & NIST_27900 & $0.00 \mathrm{E}+00$ & $0.00 \mathrm{E}+00$ & KNSTD & 20193 & 1801 & 23573 & 672 & ED-SUERC \\
\hline FLO-10-CJF & -78 & -85 & 1281 & ant & 5 & 2.65 & 0.985 & 0 & $1.00 \mathrm{E}+07$ & $2.61 \mathrm{E}+05$ & NIST_27900 & $0.00 \mathrm{E}+00$ & $0.00 \mathrm{E}+00$ & KNSTD & 659768 & 70868 & 794193 & 33072 & ED-SUERC \\
\hline FLO-15-CJF & -78 & -85 & 1309 & ant & 5 & 2.65 & 0.985 & 0 & $5.62 \mathrm{E}+06$ & $7.71 \mathrm{E}+04$ & NIST_27900 & $0.00 \mathrm{E}+00$ & $0.00 \mathrm{E}+00$ & KNSTD & 333883 & 31952 & 395275 & 11240 & ED-SUERC \\
\hline FLO-03-CJF & -78 & -84 & 1352 & ant & 5 & 2.65 & 0.985 & 0 & $1.05 \mathrm{E}+06$ & $1.98 \mathrm{E}+04$ & NIST_27900 & $0.00 \mathrm{E}+00$ & $0.00 \mathrm{E}+00$ & KNSTD & 56332 & 5078 & 65865 & 1926 & ED-SUERC \\
\hline FLO-04-CJF & -78 & -84 & 1335 & ant & 5 & 2.65 & 0.985 & 0 & $2.72 \mathrm{E}+06$ & $5.71 \mathrm{E}+04$ & NIST_27900 & $0.00 \mathrm{E}+00$ & $0.00 \mathrm{E}+00$ & KNSTD & 151396 & 14054 & 177744 & 5619 & ED-SUERC \\
\hline FLO-19-CJF & -78 & -84 & 1336 & ant & 5 & 2.65 & 0.985 & 0 & $7.62 \mathrm{E}+05$ & $1.49 \mathrm{E}+04$ & NIST_27900 & $0.00 \mathrm{E}+00$ & $0.00 \mathrm{E}+00$ & KNSTD & 41217 & 3708 & 48160 & 1426 & ED-SUERC \\
\hline FLO-20-CJF & -78 & -84 & 1335 & ant & 5 & 2.65 & 0.96 & 0 & $8.94 \mathrm{E}+05$ & $1.77 \mathrm{E}+04$ & NIST_27900 & $0.00 \mathrm{E}+00$ & $0.00 \mathrm{E}+00$ & KNSTD & 49706 & 4483 & 58096 & 1733 & ED-SUERC \\
\hline FLO-17-CJF & -78 & -84 & 1335 & ant & 5 & 2.65 & 0.985 & 0 & $2.53 \mathrm{E}+05$ & $4.23 \mathrm{E}+03$ & NIST_27900 & $0.00 \mathrm{E}+00$ & $0.00 \mathrm{E}+00$ & KNSTD & 13593 & 1207 & 15865 & 437 & ED-SUERC \\
\hline FLO-01-CJF & -78 & -84 & 1357 & ant & 5 & 2.65 & 0.985 & 0 & $5.83 \mathrm{E}+06$ & $5.24 \mathrm{E}+04$ & NIST_27900 & $0.00 \mathrm{E}+00$ & $0.00 \mathrm{E}+00$ & KNSTD & 332953 & 31633 & 394185 & 10254 & ED-SUERC \\
\hline FLO-01 & -79 & -84 & 521 & ant & 8 & 2.7 & 0.996 & 0 & $4.79 \mathrm{E}+04$ & $1.99 \mathrm{E}+03$ & 07KNSTD & $0.00 \mathrm{E}+00$ & $0.00 \mathrm{E}+00$ & KNSTD & 5288 & 510 & 6012 & 283 & CAMS-LLNL \\
\hline FLO-02 & -79 & -84 & 521 & ant & 5.5 & 2.7 & 0.996 & 0 & $4.99 \mathrm{E}+04$ & $1.48 \mathrm{E}+03$ & 07KNSTD & $0.00 \mathrm{E}+00$ & $0.00 \mathrm{E}+00$ & KNSTD & 5395 & 496 & 6260 & 231 & CAMS-LLNL \\
\hline FLO- 03 & -79 & -84 & 521 & ant & 4.5 & 2.7 & 0.996 & 0 & $5.05 \mathrm{E}+04$ & $1.12 \mathrm{E}+03$ & 07KNSTD & $0.00 \mathrm{E}+00$ & $0.00 \mathrm{E}+00$ & KNSTD & 5415 & 486 & 6334 & 197 & CAMS-LLNL \\
\hline FLO-05 & -79 & -84 & 521 & ant & 6.5 & 2.7 & 0.996 & 0 & $5.00 \mathrm{E}+04$ & $1.32 \mathrm{E}+03$ & 07KNSTD & $0.00 \mathrm{E}+00$ & $0.00 \mathrm{E}+00$ & KNSTD & 5448 & 495 & 6270 & 215 & CAMS-LLNL \\
\hline FLO-06 & -79 & -84 & 515 & ant & 3 & 2.7 & 0.996 & 0 & $4.75 \mathrm{E}+04$ & $1.32 \mathrm{E}+03$ & 07KNSTD & $0.00 \mathrm{E}+00$ & $0.00 \mathrm{E}+00$ & KNSTD & 5061 & 462 & 5994 & 212 & CAMS-LLNL \\
\hline FLO-09 & -79 & -84 & 490 & ant & 3 & 2.7 & 0.996 & 0 & $5.88 \mathrm{E}+04$ & $1.78 \mathrm{E}+03$ & 07KNSTD & $0.00 \mathrm{E}+00$ & $0.00 \mathrm{E}+00$ & KNSTD & 6415 & 591 & 7596 & 283 & CAMS-LLNL \\
\hline FLO-10 & -79 & -84 & 490 & ant & 8 & 2.7 & 0.996 & 0 & $5.00 \mathrm{E}+04$ & $1.68 \mathrm{E}+03$ & 07KNSTD & $0.00 \mathrm{E}+00$ & $0.00 \mathrm{E}+00$ & KNSTD & 5685 & 530 & 6464 & 259 & CAMS-LLNL \\
\hline \multicolumn{20}{|l|}{ Union Glacier } \\
\hline UG-15 & -80 & -81 & 962 & ant & 5 & 2.7 & 0.996 & 0 & $2.50 \mathrm{E}+06$ & $6.40 \mathrm{E}+04$ & 07KNSTD & $0.00 \mathrm{E}+00$ & $0.00 \mathrm{E}+00$ & KNSTD & 190113 & 18058 & 223025 & 7926 & CAMS-LLNL \\
\hline UG-16 & -80 & -81 & 938 & ant & 5 & 2.7 & 0.996 & 0 & $5.31 \mathrm{E}+05$ & $1.00 \mathrm{E}+04$ & 07KNSTD & $0.00 \mathrm{E}+00$ & $0.00 \mathrm{E}+00$ & KNSTD & 39660 & 3560 & 46296 & 1348 & CAMS-LLNL \\
\hline UG-19 & -80 & -81 & 911 & ant & 5 & 2.7 & 0.996 & 0 & $8.80 \mathrm{E}+05$ & $1.65 \mathrm{E}+04$ & 07KNSTD & $0.00 \mathrm{E}+00$ & $0.00 \mathrm{E}+00$ & KNSTD & 67748 & 6123 & 79187 & 2320 & CAMS-LLNL \\
\hline UG-24 & -80 & -81 & 857 & ant & 5 & 2.7 & 0.996 & 0 & $4.51 \mathrm{E}+05$ & $8.42 \mathrm{E}+03$ & 07KNSTD & $0.00 \mathrm{E}+00$ & $0.00 \mathrm{E}+00$ & KNSTD & 36072 & 3234 & 42153 & 1221 & CAMS-LLNL \\
\hline UG-27 & -80 & -81 & 839 & ant & 5 & 2.7 & 0.996 & 0 & $4.16 \mathrm{E}+05$ & $7.81 \mathrm{E}+03$ & 07KNSTD & $0.00 \mathrm{E}+00$ & $0.00 \mathrm{E}+00$ & KNSTD & 33803 & 3029 & 39475 & 1146 & CAMS-LLNL \\
\hline UG-30 & -80 & -81 & 800 & ant & 5 & 2.7 & 0.996 & 0 & $1.97 \mathrm{E}+05$ & $6.23 \mathrm{E}+03$ & 07KNSTD & $0.00 \mathrm{E}+00$ & $0.00 \mathrm{E}+00$ & KNSTD & 16544 & 1535 & 19252 & 743 & CAMS-LLNL \\
\hline
\end{tabular}

${ }^{\mathrm{a}}$ Ratio of the production rate at the shielded site to that for a $2 \pi$ surface at the same location calculated using the CRONUS-Earth geometric shielding calculator version 1.1 .

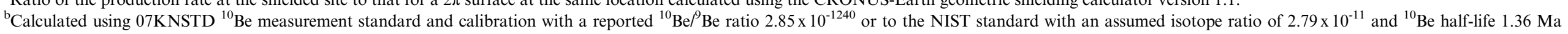
(Chmeleff et al. 2010, Korschinek et al. 2010).

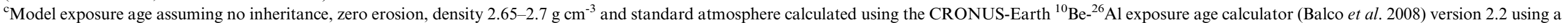
constant production rate model and scaling scheme for spallation of Lal (1991)/Stone (2000). Ages based upon global production rate $\left(\mathrm{P}_{\text {global }}\right)$ and New Zealand production rate $\left(\mathrm{P}_{\mathrm{NZ}}\right)$ accordingly.

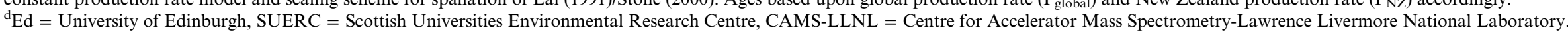


Hillenbrand et al. 2014). Whilst the existing marine chronology is open to interpretation, including possible reworking and the potentially significant changes in Antarctic marine radiocarbon reservoir effect over time (Hillenbrand et al. 2014), the ages are internally coherent, suggesting that they provide reliable constraints on the gradual retreat of the grounding line across the Weddell Sea. An important constraint from the outer continental shelf records grounding line retreat, and suggests open water conditions were established by c. $18.1 \mathrm{ka}$ (Hillenbrand et al. 2014). Two further reliable critical constraints exist close to the sills of the extensive Thiel and Rutford cross-shelf troughs in the Weddell Sea. These record retreat in the eastern Weddell Sea at the head of the Thiel Trough before $c .8 .3 \mathrm{ka}$, and at the head of the Rutford Trough in the western Weddell Sea at c. $5.3 \mathrm{ka}$ (Fig. 1) (Hillenbrand et al. 2014).

\section{Terrestrial geochronological constraints}

Although the available marine radiocarbon data is sparse, a more comprehensive terrestrial record of ice stream surface changes is recorded on exposed mountains in the catchments of the Rutford and Institute ice streams. This study combines new ${ }^{10} \mathrm{Be}$ and in situ ${ }^{14} \mathrm{C}$ data that record changes of the Rutford Ice Stream with published in situ ${ }^{10} \mathrm{Be}$ and ${ }^{26} \mathrm{Al}$ cosmogenic isotope data from the catchment of the Institute Ice Stream (Bentley et al. 2010, Fogwill et al. 2012). Terrestrial ice surface elevations through time are constructed by measuring cosmogenic nuclides in erratics glacially transported from sites located in the catchments of the Rutford Ice Stream and Institute Ice Stream, as suggested by our LGM ice sheet flow model (Fig. 2). Glacial erratics sampled from steep exposed bedrock surfaces, in the Flower Hills, Union Glacier, and the Patriot and Marble hills (Bentley et al. 2010, Fogwill et al. 2012) (Fig. 1), serve as 'dipsticks' that allow us to reconstruct past surface elevation changes in the catchments of the ice streams since the LGM.

Samples were reduced to pure quartz at the University of Edinburgh cosmogenic nuclide laboratory and Lawrence Livermore National Laboratories Center for Accelerator Mass Spectrometry (LLNL-CAMS) following standard procedures (Kohl \& Nishiizumi 1992, Ivy-Ochs 1996, Stone 2004). The ${ }^{10} \mathrm{Be}$ ratios were measured by the AMS facility at LLNL and the Scottish Universities Environmental Research Centre (SUERC) (Xu et al. 2010). Measurements were standardized to the NIST SRM-4325 Be standard material with a revised nominal ${ }^{10} \mathrm{Be} /{ }^{9} \mathrm{Be}$ ratio of $2.79 \times 10^{-11}$ (Nishiizumi et al. 2007). Samples were corrected for the number of ${ }^{10} \mathrm{Be}$ atoms in their associated blanks. Blanks were spiked with $250 \mu \mathrm{g}{ }^{9} \mathrm{Be}$ carrier (Edinburgh) and $474 \mu \mathrm{g}{ }^{9} \mathrm{Be}$ (LLNL-CAMS). The corresponding combined process and carrier blanks ${ }^{10} \mathrm{Be} /{ }^{9} \mathrm{Be}$ ratios range between 1.6-5.47 $\times 10^{-15}$. Sample and blank ${ }^{10} \mathrm{Be} /{ }^{9} \mathrm{Be}$ analytical uncertainties and a $2.5 \%$ carrier addition uncertainty are propagated into the $1 \sigma$ analytical uncertainty for nuclide concentrations.

A version of the CRONUS-Earth online age calculator was used to determine the ${ }^{10} \mathrm{Be}$ exposure ages (Balco et al. 2008), implementing the New Zealand ${ }^{10} \mathrm{Be}$ production rate calibration dataset (Putnam et al. 2010), that uses the recently revised ${ }^{10} \mathrm{Be}$ half-life $(1.387 \mathrm{Ma})$ (Chmeleff et al. 2010, Korschinek et al. 2010), and Be isotope ratio standardization of Nishiizumi (Nishiizumi et al. 2007). The use of this revised production rate and half-life change impact the apparent exposure ages, causing them to increase by c. $12 \%$ from those previously published (Bentley et al. 2010, Fogwill et al. 2012) (Table I). Choice of production rate model and scaling is often a pragmatic one and is an ongoing subject of debate. Here the New Zealand calibration dataset was used to allow comparison with other recent Antarctic studies and in the absence of an Antarctic production rate calibration site. Exposure ages are reported based on the Lal/Stone scaling model for Antarctica; using the same calibration dataset, ages differ by $2-4 \%$ depending on the choice of scaling model (Balco et al. 2008). The calculator uses sample thickness and density to standardize nuclide concentrations to the rock surface. The whole rock density is assumed to be $2.65-2.7 \mathrm{~g} \mathrm{~cm}^{-3}$. No correction for periodic snow cover or for rock-surface erosion was included, as both of which are assumed to be negligible in these sites. An erosion rate of $0.0002 \mathrm{~cm} \mathrm{yr}^{-1}$ increases ages by $c .2 \%$.

Uniquely, this study also takes advantage of recent technological developments in the extraction and measurement of in situ radiocarbon $\left({ }^{14} \mathrm{C}\right)$ from quartz (Hippe et al. 2009, 2013), a cosmogenic nuclide with a considerably shorter half-life than that of ${ }^{10} \mathrm{Be}$ $\left({ }^{10} \mathrm{Be}=1.36 \times 10^{3} \mathrm{kyr},{ }^{14} \mathrm{C}=5.73 \mathrm{kyr}\right)$. As the relatively short half-life of ${ }^{14} \mathrm{C}$ means that in situ ${ }^{14} \mathrm{C}$ acquired on exposure during interglacials decays if the sample is covered by ice during a subsequent glacial, the apparent ${ }^{14} \mathrm{C}$ age reflects the true minimum exposure age of the sample. Crucially, the disparity between the ${ }^{10} \mathrm{Be}$ and ${ }^{14} \mathrm{C}$ data allows the potential influence of prior exposure or recycling in this setting to be assessed (Lifton et al. 2001, White et al. 2011).

In situ ${ }^{14} \mathrm{C}$ extraction was performed at ETH Zürich following a modified protocol (Hippe et al. 2009, 2013). Quartz aliquots of $c .5 \mathrm{~g}$ were preheated at $c .700^{\circ} \mathrm{C}$ to remove atmospheric ${ }^{14} \mathrm{C}$ contamination followed by the extraction of in situ ${ }^{14} \mathrm{C}$ during heating to $1550-1600^{\circ} \mathrm{C}$ for $2 \times 2$ hours. The collected $\mathrm{CO}_{2}$ gas was split into two samples before AMS measurement due to large gas amounts. Samples were then measured with the MICADAS AMS system using the gas ion source (Ruff et al. 2007, Synal et al. 2007, Wacker et al. 2010). The number of ${ }^{14} \mathrm{C}$ atoms obtained for both splits 
were summed prior to subtraction of the long-term average processing blank of $(3.15 \pm 1.19) \times 10^{4}{ }^{14} \mathrm{C}$ atoms ( \pm 1 standard deviation, $n=24$ ).

\section{${ }^{14} \mathrm{Cl}^{10} \mathrm{Be}$ multi-isotope analysis}

For this study, in situ ${ }^{14} \mathrm{C}$ exposure ages were calculated with a sea level, high latitude (SLHL) spallogenic production rate of $11.40 \pm 0.9$ at g$^{-1} \mathrm{y}^{-1}$ (Schimmelpfennig et al. 2014). As with ${ }^{10} \mathrm{Be}$, the production rate was scaled to altitude and latitude according to the scaling scheme of Lal/Stone. The contribution due to muon production was calculated using the freely accessible MATLAB code of the CRONUS-Earth online calculator (http.//hess.ess. washington.edu/math/al_be_v2/P_mu_total) (Balco et al. 2008). In order to allow muon scaling for in situ ${ }^{14} \mathrm{C}$, parameters were adjusted based on the cross sections for ${ }^{14} \mathrm{C}$ (Heisinger et al. 2002a, 2002b), and corrections for sample thickness and topographical shielding were applied on spallogenic production only.

Combined ${ }^{14} \mathrm{C}$ and ${ }^{10} \mathrm{Be}$ analysis is applied to sites in the Flower Hills and Union Glacier (in the catchment of the Rutford Ice Stream) which display a high percentage of anomalously 'old' apparent ${ }^{10} \mathrm{Be}$ exposure ages (Table I). The disparity between the in situ ${ }^{14} \mathrm{C}$ and ${ }^{10} \mathrm{Be}$ data demonstrates that the samples have experienced a complicated exposure history, suggesting either that the cosmogenic nuclide inventories of the erratics were not fully reset by glacial erosion prior to deposition, or that following initial deposition they underwent periods of exposure at different altitudes and/or cover by cold-based ice (White et al. 2011). Using the measured concentrations of both ${ }^{10} \mathrm{Be}$ and ${ }^{14} \mathrm{C}$, an iterative model was constructed to calculate the maximum and minimum periods of ice cover each sample could have undergone to explain the differing nuclide concentrations. These periods were then compared with the equivalent periods of ice cover implied by the eustatic sea level data, following a similar approach to studies of the Fennoscandian Ice Sheet (Fabel et al. 2002).

Whilst only a one-way test, this assumes that the erratics experienced periods of ice cover subsequent to initial deposition at any point when sea level was lower (and ice volume greater) than it was at the point of re-exposure given by the ${ }^{14} \mathrm{C}$ apparent exposure age (Table II). Using the measured, minimum and maximum ${ }^{14} \mathrm{C}$ exposure ages of each sample (given by the external errors within the process, to allow for comparability with the independently dated sea level curve), three scenarios for each sample were created, under which all samples apart from UG-27 are shown to have experienced one period of extended ice cover following initial deposition, followed by subsequent re-exposure (Fig. 3). Sample UG-27 has a complex nuclide inventory, possibly reconcilable by either a single or multiple pre-exposure event at a higher altitude than 

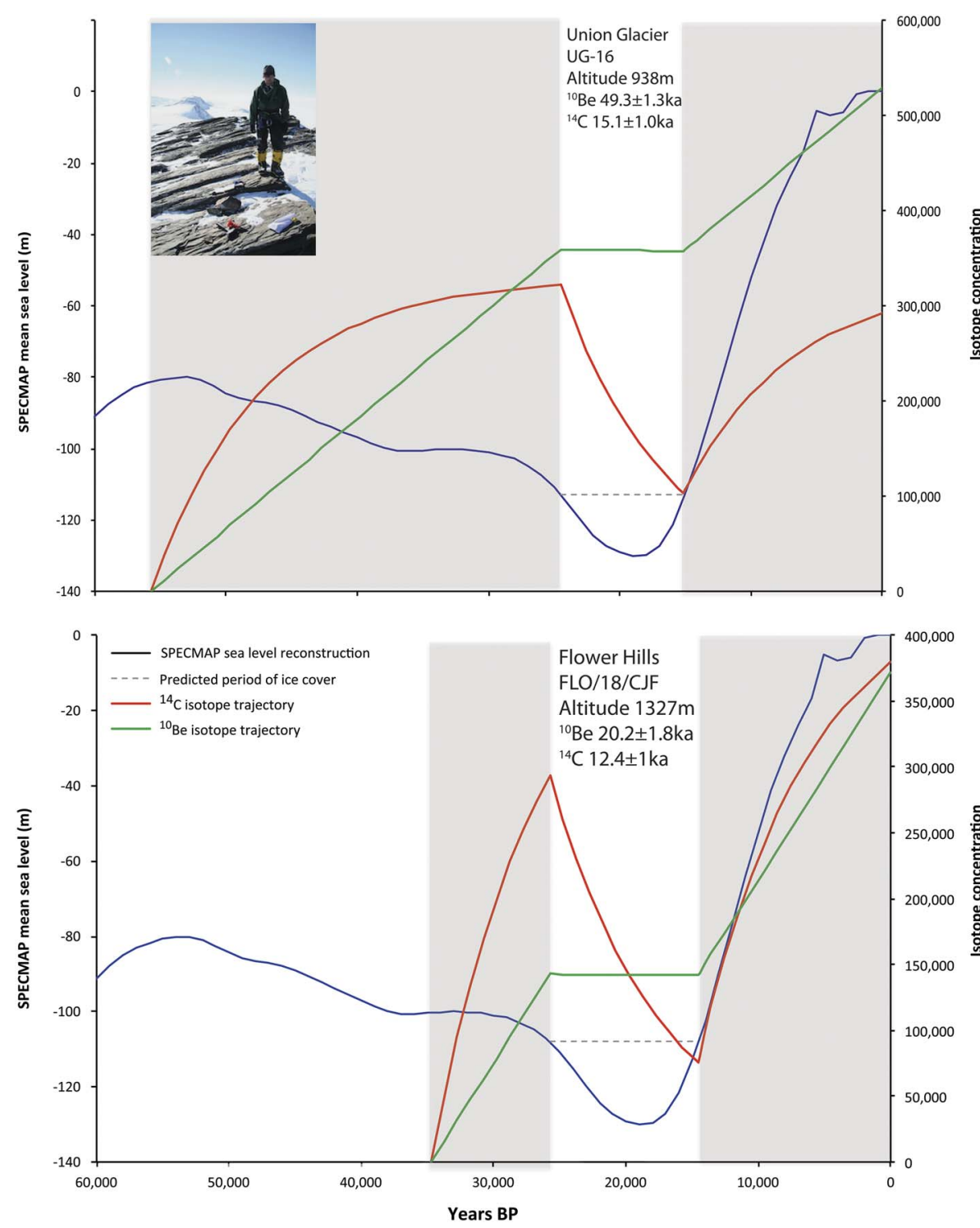

Fig. 3. Modelled relationship between ${ }^{10} \mathrm{Be} /{ }^{14} \mathrm{C}$ isotope concentrations, time and sea level used as a proxy for global ice volume (Imbrie \& McIntyre 2006) for samples FLO/18/ CJF and UG16. Proposed periods of sample exposure are defined by the grey boxes. The altitude and apparent exposure ages based upon the measured ${ }^{10} \mathrm{Be}$ and ${ }^{14} \mathrm{C}$ inventories of the samples are noted. The inset photo shows sample FLO/18/CJF, a quartzite erratic on striated agrilite bedrock typical of the samples analysed. present. For the remaining samples, a scenario is identified that agrees with the periods of ice cover stipulated by the cosmogenic nuclide data and the sea level reconstruction: a single extended period of ice cover following initial deposition, and subsequent re-exposure during the last deglaciation (Fig. 3). This suggests that the disparity between the ${ }^{14} \mathrm{C} /{ }^{10} \mathrm{Be}$ is probably a result of cover by cold-based ice, and demonstrates that the use of another isotope paired alongside ${ }^{14} \mathrm{C}$ can provide insights into the depositional history of the samples, allowing for a more confident interpretation of the surface trajectory of the Rutford Ice Stream.

\section{Results}

To examine the dynamic glaciological changes recorded from our geological reconstruction, firstly the changes to geometry and ice flow pattern triggered by post-LGM increases in oceanic heat flux and sea level were assessed. The patterns of ice flow predicted by the model under this scenario are shown in Fig. 2. The initial response of the LGM ice sheet to ocean and atmospheric forcing is depicted in Fig. 2a, and is marked by almost uniform grounding line retreat across the WSE, coupled with high discharge rates through all of the major cross-shelf troughs. The predicted ice sheet surface remains above $1300 \mathrm{~m}$ in the catchments of both ice streams.

Figure $2 \mathrm{~b}$ shows the rapid increase in predicted ice flux in response to the prescribed ocean forcing, with acceleration of flow at the marine margins and concomitant drawdown of the ice sheet surface in the WSE. Although ice flux is greatest at the head of the deep Thiel Trough and its tributaries, ice from both the Rutford Ice Stream and Institute Ice Stream continue to discharge through the Rutford Trough and the extended Evans Trough on the western side of the WSE. Due to the location of the two ice 


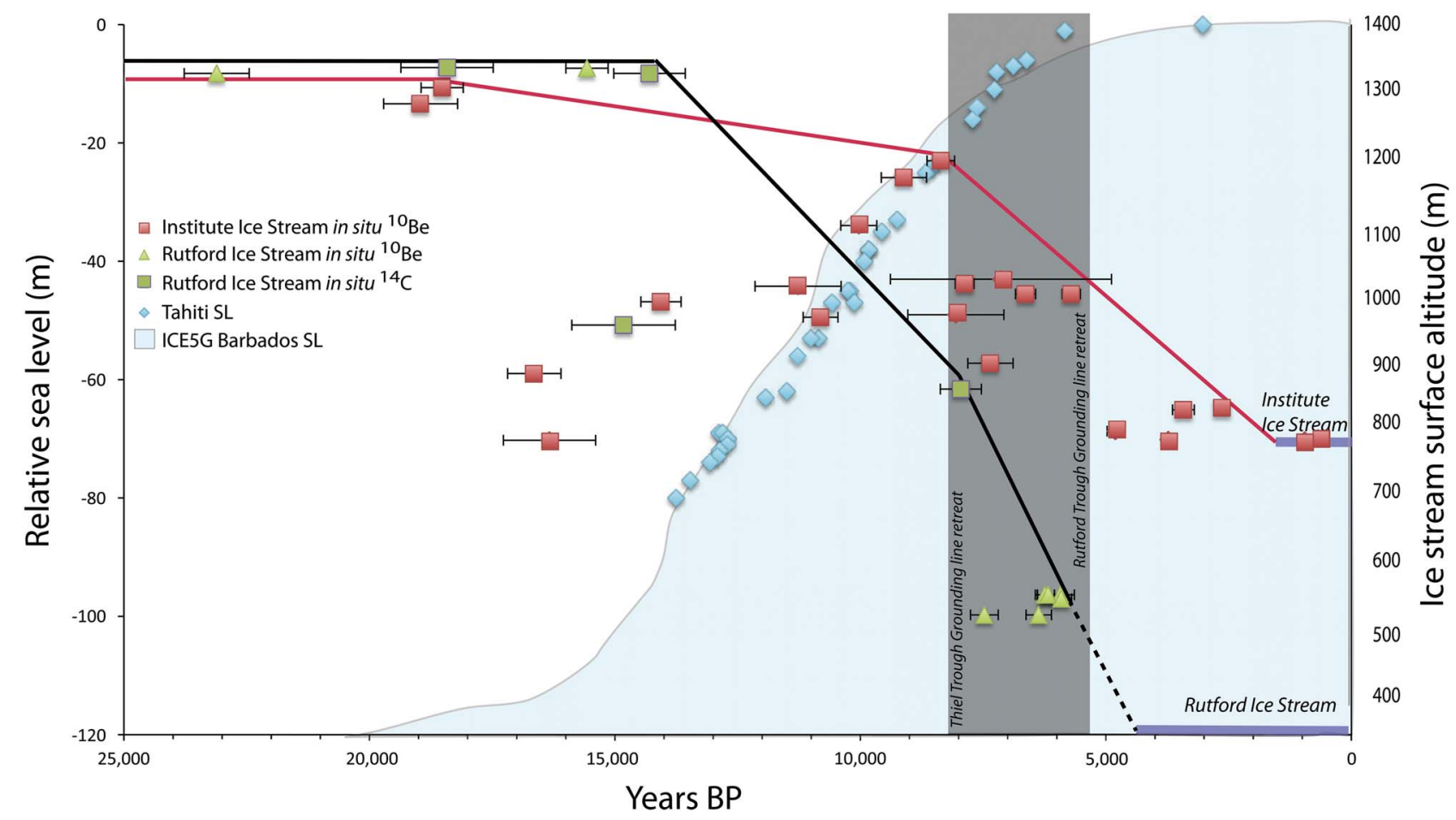

Fig. 4. Reconstructed ice stream trajectories over the last 25000 years from terrestrial cosmogenic nuclides in glacially transported erratics (in situ ${ }^{14} \mathrm{C}$ and ${ }^{10} \mathrm{Be} \pm 1$ standard deviation). The profiles of the Rutford and Institute ice streams are shown in green and red, respectively. The grey column defines the timing of inner continental shelf deglaciation of the Thiel Trough and the Rutford Trough, based upon the available calibrated marine ${ }^{14} \mathrm{C}$ constraints (Hillenbrand et al. 2012, 2014), reflecting the proposed period of ice stream capture of the Institute Ice Stream by the Thiel Trough. For comparison, global relative sea level rise reconstructed from Tahiti (Bard et al. 1996, Bard 2003) and Barbados (Peltier \& Fairbanks 2006) are plotted.

streams relative to the grounding line, this leads to the start of a marked and rapid drawdown of the Rutford Ice Stream at this time when compared to the Institute, reflected in rapid altitudinal change in the catchment of the Rutford Ice Stream after 15 ka (Fig. 2b).

Under continued oceanic forcing, grounding line retreat in the WSE becomes markedly asymmetric, with faster retreat taking place in the Thiel Trough (eastern WSE) than in the west (Fig. 2c upper panel). Consequently, the inland ice sheet surface gradient switches from its formerly north-easterly direction to a more east-southeasterly direction, with the effect that ice discharging in the Institute and Möller ice streams is diverted towards the Thiel Trough (Fig. 2c lower panel). Thus at this point, drainage of these neighbouring ice streams becomes governed by the locations of two separate grounding lines c. $300 \mathrm{~km}$ apart. Their behaviour is thus decoupled from one another, allowing independent thinning trajectories during deglaciation.

\section{Discussion}

The geological reconstructions presented here mirror the results of the ice sheet model simulation, and provide a chronological framework to examine the physical effects of grounding line retreat away from the marine margin. The results demonstrate that the surface of the Rutford Ice Stream and Institute Ice Stream exceeded $1300 \mathrm{~m}$ in altitude at the LGM, buttressed by grounded ice in the Weddell Sea (Fig. 4, see Tables I \& II for details). Geologically this upper limit of the ice stream surfaces is defined based on the absence of any apparently 'young' (post-LGM) exposure ages above this altitude (Bentley et al. 2010, 2011, Clark 2011), and the presence of locally derived LGM-age ice in the Patriot Hills, as demonstrated by recent analysis of the exposed blue ice in the Institute Ice Stream catchment (Turney et al. 2013). Based upon this interpretation it is apparent that the Rutford and Institute ice streams maintained their LGM surface profiles until $16 \mathrm{ka}$, away from the marine margins of the retreating grounding line despite rising sea level and regional ocean circulation and temperature changes (Fig. 4). This is supported by comparison of the ice stream trajectories with post-LGM eustatic global sea level, which suggest a delayed response of both the Rutford and the Institute ice streams to global sea level rise.

After c. $16 \mathrm{ka}$ the results suggest that the surface trajectories of the two ice streams began to diverge (Fig. 4). 
Initially the Institute Ice Stream thinned slowly, dropping in elevation by only $100 \mathrm{~m}$ between $c .20$ and $c .8 .5 \mathrm{ka}$. Subsequently the rate of decay increased markedly after c. $8.5 \mathrm{ka}$, thinning $380 \mathrm{~m}$ in $c .6000$ years, to reach the present ice sheet surface elevation by $c .2 \mathrm{ka}$, supporting interpretations of the regional isostatic response derived from GPS constraints (Argus et al. 2011).

The Institute Ice Stream's surface trajectory contrasts with that of the Rutford Ice Stream, which maintained an altitude of over $1300 \mathrm{~m}$ until decay was initiated at c. $14.5 \mathrm{ka}$ (Fig. 4). At this time, the Rutford apparently decayed rapidly, thinning by $c .900 \mathrm{~m}$ between $c .14 .5 \mathrm{ka}$ and $c .6 \mathrm{ka}$. Although the trajectory of the Rutford Ice Stream after $c$. $6 \mathrm{ka}$ between the lower sample sites $(520-490 \mathrm{~m})$ and the present ice stream surface altitude cannot be fully defined, a lack of geomorphological evidence on the steep slopes between these altitudes suggests that the downward thinning trajectory of the Rutford Ice Stream continued in response to grounding line retreat across the inner shelf of the western Weddell Sea at c. $5.3 \mathrm{ka}$ (Fig. 1) (Hillenbrand et al. 2012).

Whilst the terrestrial geological reconstruction presented here is unable to rule out if this thinning of either the Rutford or Institute ice streams continued below present levels into the late Holocene this is unlikely based upon independent evidence from the region which suggests relative stability since c. 4 ka (Argus et al. 2011, Turney et al. 2013). The combination of stability of ice in the catchment of the Institute Ice Stream, and the regional isostatic uplift signal argues that there was no significant recent loss (and subsequent rapid re-expansion) of the deep basins upstream of the present grounding line, as has been suggested from the interpretation of regional airborne RES (Siegert et al. 2013).

Together, the ice sheet model simulations and geological reconstruction presented in the this study demonstrate asymmetry in ice dynamics between the Rutford Ice Stream and Institute Ice Stream during the last glacialinterglacial transition, which realigns during the mid Holocene between $c .8 .3$ and $5.3 \mathrm{ka}$. This reflects a regional-scale diversion of ice discharge in the Institute Ice Stream due to ice stream capture by the Thiel Trough palaeo ice stream after grounding line retreat between c. 8.3 and $5.3 \mathrm{ka}$, which impacted regional mass balance in this sector of the WAIS (Fig. 2c). Significantly, this analysis shows that both the Institute and Möller ice streams are susceptible to capture. Additionally, this interpretation corroborates a recent interpretation of marine geophysical evidence which suggests that the Foundation Ice Stream may also be affected by ice stream flow diversion (Larter et al. 2012). All other modelled outlets in the western WSE continue to drain through Rutford Trough, regardless of grounding line position or dynamics.

This threshold-controlled behaviour of the Institute and Möller ice streams is probably a consequence of their central position between the two major cross-shelf troughs, implying that subglacial topography underlying the ice stream does not significantly restrict flow to a particular route. This has important ramifications for future ice sheet dynamics in the WSE, suggesting that predicted twenty-first century ocean warming in the Thiel Trough (Fogwill et al. 2012, Hellmer et al. 2014) could re-instigate capture of these ice streams. Such a divergence may lead to a marked response due to the deep subglacial basins that exist upstream of the grounding lines (Ross et al. 2012). Whilst previous studies have highlighted switches in ice stream direction using different approaches, including marine geophysical techniques (e.g. Larter et al. 2012), glaciological investigations (Conway et al. 2002) and ice sheet modelling studies (Payne 1999), none have been independently verified by the combined ice sheet modelling and empirical geological approach as described here.

Whilst surface exposure ages in the eastern Weddell Sea suggest that the modelled ice sheet may be too thick in this region at the LGM (Golledge et al. 2012), the limited thickening implied by empirical terrestrial data (Fogwill et al. 2004, Hein et al. 2011), coupled with the greatly advanced grounding line position interpreted from marine geological data (Hillenbrand et al. 2014), can only be reconciled with a surface slope of the LGM grounded ice sheet that is similar to that of the present ice shelf. This suggests an extremely low basal shear stress $(<15 \mathrm{kPa})$, and it is acknowledged that this disagreement with the observations requires further investigation.

In summary, the asynchronous response of the Rutford Ice Stream and Institute Ice Stream to post-LGM ice sheet reconfiguration reflects the combination of streaming ice flow and spatially variable bathymetric controls on the inner continental shelf, which caused tipping points to be passed during deglaciation, leading to jumps between stable flow patterns. Importantly, both of these major arteries of the WAIS show a remarkable delay in their response to external forcing, particularly sea level, implying that other internal mechanisms are at work. When aligned to marine records, these data reveal that the onset and rate of deglaciation of the Rutford Ice Stream and Institute Ice Stream are controlled independently by grounding line retreat within the Thiel and Rutford troughs, respectively. These findings support recent inference from marine and terrestrial geophysical surveys, which suggest that during deglaciation icedrainage pathways in the WSE may well have differed from those observed today (Larter et al. 2012, Stolldorf et al. 2012, Siegert et al. 2013). Importantly, these reconstructions, together with independent constraints (Argus et al. 2011, Turney et al. 2013), do not suggest that the Institute Ice Stream has undergone significant drawdown during the late Holocene or subsequent significant re-expansion (Siegert et al. 2013); rather, the 
results suggest that late Holocene ice stream reconfiguration of the Weddell Sea was driven by spatially variable ice flux at the marine margin, which modulated the direction of individual ice streams of the WAIS during the early Holocene.

\section{Conclusions}

The data presented here have demonstrated that two major ice streams of the WSE had an asynchronous response to ocean-forced grounding line retreat. To understand the mechanism for these divergent trends, flow changes predicted by our high-resolution ice sheet simulation, which simulated grounding line retreat in the WSE, were analysed. The decoupling of the surface trajectories of the two ice streams was driven by differences in the rate of grounding line retreat across the WSE, resulting in the Institute Ice Stream switching direction by more than $60^{\circ}$ and discharging ice into the Thiel Trough during the early Holocene, rather than the Rutford Trough as it does at present. The new terrestrial geochronological constraints (in situ ${ }^{14} \mathrm{C}$ and ${ }^{10} \mathrm{Be}$ ) reveal that although these two adjacent ice streams exhibited similar surface geometries at the end of the LGM, the pattern of ice surface lowering contrasted markedly after this, with asynchronous thinning trajectories during the late to mid Holocene.

These findings highlight that spatial variability in ice flow can trigger marked changes in the pattern, flux and flow direction of extensive ice streams on millennial timescales, markedly changing regional ice sheet mass balance. A detailed understanding of these abrupt diversions is critical to improve predictions for future WAIS stability in light of the sensitivity of the Institute Ice Stream to marine ice sheet instability today, with its present grounding line below mean sea level at the head of an extensive subglacial trough. Given this evidence of potential flow switches in the WSE, and in light of projected twenty-first century regional ocean warming in the Thiel Trough, the ability to predict these abrupt and extensive diversions is a priority within the glaciological community, achievable only through the coupling of high-resolution ice sheet and ocean models.

\section{Acknowledgements}

This research was supported by the Australian Research Council (FL100100195, FT1201000004 and LP120200724), and UK National Environmental Research Council (AFI 05/03). Fieldwork was supported by Antarctic Logistics and Expeditions and the British Antarctic Survey. NRG gratefully acknowledges support from Victoria University of Wellington and GNS Science. CJF wishes to thank Dr Neil Ross for helpful discussions, Prof David Sugden for instigating this work, and Dr Claus-Dieter Hillenbrand and two anonymous reviewers for their helpful comments.

\section{Author contributions}

CJF, CSMT and NRG conceived the project. NRG undertook the ice sheet modelling experiments and designed the model simulation with CJF. CJF and DHR analysed the ${ }^{10} \mathrm{Be}$ samples at the University of Edinburgh and Lawrence Livermore National Laboratory, respectively. KH, RW and $\mathrm{LW}$ developed the ${ }^{14} \mathrm{C}$ extraction line and gas measurement techniques at ETH Zürich. CJF, DHR, KH, EBR, RSJ and RW analysed the ${ }^{10} \mathrm{Be} /{ }^{14} \mathrm{C}$ data interpretation. All authors discussed the results and implications, and commented on the manuscript at all stages.

\section{References}

Argus, D.F., Blewitt, G., Peltier, W.R. \& Kreemer, C. 2011. Rise of the Ellsworth mountains and parts of the East Antarctic coast observed with GPS. Geophysical Research Letters, 38, 10.1029/ 2011 GL048025.

Balco, G., Stone, J.O., Lifton, N.A. \& Dunai, T.J. 2008. A complete and easily accessible means of calculating surface exposure ages or erosion rates from ${ }^{10} \mathrm{Be}$ and ${ }^{26} \mathrm{Al}$ measurements. Quaternary Geochronology, 3, 174-195.

Bamber, J.L., Riva, R.E.M., Vermeersen, B.L.A. \& Le Broce, A.M. 2009. Reassessment of the potential sea-level rise from a collapse of the West Antarctic Ice Sheet. Science, 324, 901-903.

BARD, E. 2003. Tahiti deglacial relative sea level reconstruction. IGBP PAGES/World Data Center for Paleoclimatology Data Contribution Series \#2003-028. Boulder, CO: NOAA/NGDC Paleoclimatology Program.

Bard, E., Hamelin, B., Arnold, M., Montaggioni, L., Cabioch, G., Faure, G. \& Rougerie, F. 1996. Deglacial sea level record from Tahiti corals and the timing of meltwater discharge. Nature, 382, 241-244.

Bentley, M.J., Fogwill, C.J., Le Broce, A.M., Hubbard, A.L., Sugden, D.E., Dunai, T.J. \& Freeman, S.P.H.T. 2010. Deglacial history of the West Antarctic Ice Sheet in the Weddell Sea embayment: constraints on past ice volume change. Geology, 38, $411-414$.

Bentley, M.J., Sugden, D.E., Fogwill, C.J., Le BrocQ, A.M., Hubbard, A.L., Dunai, T.J. \& Freeman, S.P.H.T. 2011. Deglacial history of the West Antarctic Ice Sheet in the Weddell Sea embayment: constraints on past ice volume change: REPLY. Geology, 39, 10.1130/G32140Y.1.

ChmelefF, J., von Blanckenburg, F., Kossert, K. \& Jakob, D. 2010. Determination of the ${ }^{10} \mathrm{Be}$ half-life by multicollector ICP-MS and liquid scintillation counting. Nuclear Instruments and Methods in Physics Research, B268, 192-199.

Clark, P.U. 2011. Deglacial history of the West Antarctic Ice Sheet in the Weddell Sea embayment: constraints on past ice volume change: COMMENT. Geology, 39, 10.1130/G31533C.1.

Comiso, J. 2000. Variability and trends in Antarctic surface temperatures from in situ and satellite infrared measurements. Journal of Climate, 13, 1674-1696.

Conway, H., Catania, G., Raymond, C.F., Gades, A.M., Scambos, T.A. \& Engelhardt, H. 2002. Switch of flow direction in an Antarctic ice stream. Nature, 419, 465-467.

Cuffey, K.M. 2011. Antarctic ice flow revealed. Science, 333, 1386-1387. 
Fabel, D., Stroeven, A.P., Harbor, J., Kleman, J., Elmore, D. \& FINK, D. 2002. Landscape preservation under Fennoscandian ice sheets determined from in situ produced Be-10 and Al-26. Earth and Planetary Science Letters, 201, 397-406.

Fogwill, C.J., Hein, A.S., Bentley, M.J. \& Sugden, D.E. 2012. Do blue-ice moraines in the Heritage Range show the West Antarctic Ice Sheet survived the last interglacial? Palaeogeography, Palaeoclimatology, Palaeoecology, 335, 61-70.

Fogwill, C.J., Bentley, M.J., Sugden, D.E., Kerr, A.R. \& Kubik, P.W. 2004. Cosmogenic nuclides ${ }^{10} \mathrm{Be}$ and ${ }^{26} \mathrm{Al}$ imply limited Antarctic ice sheet thickening and low erosion in the Shackleton Range for $>1$ m.y. Geology, 32, 265-268.

Fogwill, C.J., Turney, C.S.M., Meissner, K.J., Golledge, N.R., Spence, P., Roberts, J.L., England, M.H., Jones, R.T. \& CARter, L. 2014. Testing the sensitivity of the East Antarctic Ice Sheet to Southern Ocean dynamics: past changes and future implications. Journal of Quaternary Science, 29, 91-98.

Golledge, N.R., Fogwill, C.J., Mackintosh, A.N. \& Buckley, K.M. 2012. Dynamics of the Last Glacial Maximum Antarctic ice-sheet and its response to ocean forcing. Proceedings of the National Academy of Sciences of the United States of America, 109, 16 052-16056.

Golledge, N.R., Levy, R.H., McKay, R.M., Fogwill, C.J., White, D.A., Graham, A.G.C., Smith, J.A., Hillenbrand, C.-D., Licht, K.J., Denton, G.H., Ackert, R.P., MaAs, S.M. \& Hall, B.L. 2013. Glaciology and geological signature of the Last Glacial Maximum Antarctic ice sheet. Quaternary Science Reviews, 78, 225-247.

Hein, A.S., Fogwill, C.J., Sugden, D.E. \& Xu, S. 2011. Glacial/ interglacial ice-stream stability in the Weddell Sea embayment, Antarctica. Earth and Planetary Science Letters, 307, 211-221.

Heisinger, B., Lal, D., Jull, A.J.T., KubiK, P., Ivy-Ochs, S., Knie, K. $\&$ Nolte, E. 2002a. Production of selected cosmogenic radionuclides by muons. 2. Capture of negative muons. Earth and Planetary Science Letters, 200, 357-369.

Heisinger, B., Lal, D., Jull, A.J.T., Kubik, P., Ivy-Ochs, S., Neumaier, S., Knie, K., Lazarev, V. \& Nolte, E. 2002 b. Production of selected cosmogenic radionuclides by muons. 1 . Fast muons. Earth and Planetary Science Letters, 200, 345-355.

Hellmer, H.H., Kauker, F., Timmermann, R., Determann, J. \& Rae, J. 2012. Twenty-first-century warming of a large Antarctic ice-shelf cavity by a redirected coastal current. Nature, 485, 225-228.

Hillenbrand, C.D., Melles, M., Kuhn, G. \& Larter, R.D. 2012. Marine geological constraints for the grounding-line position of the Antarctic ice sheet on the southern Weddell Sea shelf at the Last Glacial Maximum. Quaternary Science Reviews, 32, 25-47.

Hillenbrand, C.D., Bentley, M.J., Stolldorf, T.D., Hein, A.S., Kuhn, G., Graham, A.G.C., Fogwill, C.J., Kristoffersen, Y., Smith, J.A., Anderson, J.B., Larter, R.D., Melles, M., Hodgson, D., Mulvaney, R. \& Sugden, D.E. 2014. Reconstruction of changes in the Weddell Sea sector of the Antarctic Ice Sheet since the Last Glacial Maximum. Quaternary Science Reviews, 10.1016/j. quascirev.2013.10.016.

Hippe, K., Kober, F., Baur, H., Ruff, M., Wacker, L. \& Wieler, R. 2009. The current performance of the in situ ${ }^{14} \mathrm{C}$ extraction line at ETH. Quaternary Geochronology, 4, 493-500.

Hippe, K., Kober, F., Wacker, L., FAhrni, S.M., Ivy-Ochs, S., Akçar, N., SChluchter, C. \& Wieler, R. 2013. An update on in situ cosmogenic ${ }^{14} \mathrm{C}$ analysis at ETH Zürich. Nuclear Instruments and Methods in Physics Research Section, B294, 81-86.

Imbrie, J.D. \& McIntyre, A. 2006. SPECMAP time scale developed by Imbrie et al. 1984 based on normalized planktonic records (normalized O-18 vs time, specmap.017). Earth System Science Data, 10.15941PANGAEA.441706

Ivy-Ochs, S. 1996. The dating of rock surfaces using in situ produced ${ }^{10} \mathrm{Be}$, ${ }^{26} \mathrm{Al}$ and ${ }^{36} \mathrm{Cl}$, with examples from Antarctica and the Swiss Alps. $\mathrm{PhD}$ thesis, Zurich ETH, 197 pp. [Unpublished].
Kohl, C.P. \& Nishizzumi, K. 1992. Chemical isolation of quartz for measurement of in-situ produced cosmogenic nuclides. Geochimica et Cosmochimica Acta, 56, 3583-3587.

Korschinek, G., Bergmaier, A., Faestermann, T., et al. 2010. A new value for the half-life of ${ }^{10} \mathrm{Be}$ by heavy-ion elastic recoil detection and liquid scintillation counting. Nuclear Instruments and Methods in Physics Research Section, B268, 187-191.

Larter, R.D., Graham, A.G.C., Hillenbrand, C.-D., Smith, J.A. \& Gales, J.A. 2012. Late Quaternary grounded ice extent in the Filchner Trough, Weddell Sea, Antarctica. new marine geophysical evidence. Quaternary Science Reviews, 53, 111-122.

Le Broce, A., Payne, A. \& Vieli, A. 2010. An improved Antarctic dataset for high resolution numerical ice sheet models (ALBMAP v1). Earth System Science Data, 2, 247-260.

Lifton, N.A., Jull, A.J.T. \& Quade, J. 2001. A new extraction technique and production rate estimate for in situ cosmogenic ${ }^{14} \mathrm{C}$ in quartz. Geochimica et Cosmochimica Acta, 65, 1953-1969.

Lisiecki, L.E. \& Raymo, M.E. 2005. A Pliocene-Pleistocene stack of 57 globally distributed benthic ${ }^{18} \mathrm{O}$ records. Paleoceanography, 20, 10.1029/2004PA001071.

Nishizumi, K., Imamura, M., Caffee, M.W., Southon, J.R., Finkel, R.C. \& McAninch, J. 2007. Absolute calibration of ${ }^{10} \mathrm{Be}$ AMS standards. Nuclear Instruments and Methods in Physics Research Section, B258, 403-413.

PAYNe, A.J. 1999. A thermomechanical model of ice flow in West Antarctica. Climate Dynamics, 15, 115-125.

Peltier, W.R. \& Fairbanks, R.G. 2006. Global glacial ice volume and Last Glacial Maximum duration from an extended Barbados sea level record. Quaternary Science Reviews, 25, 3322-3337.

Petit, J.R., Jouzel, J., Raynaud, D., et al. 1999. Climate and atmospheric history of the past 420,000 years from the Vostok ice core, Antarctica. Nature, 399, 429-436.

Putnam, A.E., Schaefer, J.M., Barrell, D.J.A., Vandergoes, M., Denton, G.H., Kaplan, M.R., Finkel, R.C., Schwartz, R., Goehring, B.M. \& Kelley, S.E. 2010. In situ cosmogenic ${ }^{10} \mathrm{Be}$ production-rate calibration from the Southern Alps, New Zealand. Quaternary Geochronology, 5, 392-409.

Rignot, E., Mouginot, J. \& Scheuchl, B. 2011. Ice flow of the Antarctic ice sheet. Science, 333, 1427-1430.

Rignot, E., Mouginot, J., Morlighem, M., Seroussi, H. \& Scheuchl, B. 2014. Widespread, rapid grounding line retreat of Pine Island, Thwaites, Smith and Kohler glaciers, West Antarctica from 1992 to 2011. Geophysical Research Letters, 41, 3502-3509.

Ross, N., Bingham, R.G., Corr, H.F.J., Ferraccioli, F., Jordan, T.A., Le Broce, A., Rippin, D.M., Young, D., Blankenship, D.D. \& Siegert, M.J. 2012. Steep reverse bed slope at the grounding line of the Weddell Sea sector in West Antarctica. Nature Geoscience, 5, 393-396.

Ruff, M., Wacker, L., Gaggeler, H.W., Suter, M., Synal, H.A. \& SzIDAT, S. 2007. A gas ion source for radiocarbon measurements at $200 \mathrm{kV}$. Radiocarbon, 49, 307-314.

Schimmelpfennig, I., Schaefer, J.M., Akçar, N., Koffman, T., Ivy-Ochs, S., Schwartz, R., Finkel, R.C., Zimmerman, S. \& SCHLÜChter, C. 2014. A chronology of Holocene and Little Ice Age glacier culminations of the Steingletscher, Central Alps, Switzerland, based on high-sensitivity beryllium-10 moraine dating. Earth and Planetary Science Letters, 393, 220-230.

Shapiro, N.M. \& Ritzwoller, M.H. 2004. Inferring surface heat flux distributions guided by a global seismic model: particular application to Antarctica. Earth and Planetary Science Letters, 223, 213-224.

Siegert, M., Ross, N., Corr, H., Kingslake, J. \& Hindmarsh, R. 2013. Late Holocene ice-flow reconfiguration in the Weddell Sea sector of West Antarctica. Quaternary Science Reviews, 78, 98-107. 
Stolldorf, T., Schenke, H.W. \& Anderson, J.B. 2012. LGM ice sheet extent in the Weddell Sea. evidence for diachronous behavior of Antarctic ice sheets. Quaternary Science Reviews, 48, 20-31.

Stone, J.O. 2004. Extraction of $\mathrm{Al}$ and Be from quartz for isotopic analysis. Seattle, WA: Cosmogenic Nuclide Laboratories at the University of Washington, $8 \mathrm{pp}$. Available at: http://depts. washington.edu/cosmolab/chem/Al-26_Be-10.pdf.

Synal, H.A., Stocker, M. \& Suter, M. 2007. MiCADAS: a new compact radiocarbon AMS system. Instruments and Methods in Physics Research, B259, 7-13.

Thomas, R.H. \& Bentley, C.R. 1978. A model for Holocene retreat of the West Antarctic Ice Sheet. Quaternary Research, 10, $150-170$.

Turney, C., Fogwill, C., van Ommen, T.D., Moy, A.D., Etheridge, D., Rubino, M., Curran, M.A.J. \& Rivera, A. 2013. Late Pleistocene and early Holocene change in the Weddell Sea: a new climate record from the Patriot Hills, Ellsworth Mountains, West Antarctica. Journal of Quaternary Science, 28, 697-704.
Van de Berg, W.J., van den Broeke, M.R., Reijmer, C.H. \& van MeijgaArd, E. 2006. Reassessment of the Antarctic surface mass balance using calibrated output of a regional atmospheric climate model. Journal of Geophysical Research - Atmospheres, 111, 10.1029/ 2005JD006495.

Wacker, L., Bonani, G., Friedrich, M., Hajdas, I., Kromer, B., Nemec, M., Ruff, M., Suter, M., Synal, H.A. \& Vockenhuber, C. 2010. MICADAS: routine and high-precision radiocarbon dating. Radiocarbon, 52, 252-262.

Weertman, J. 1974. Stability of the junction of an ice sheet and an ice shelf. Journal of Glaciology, 13, 3-11.

White, D., Fülöp, R.H., Bishop, P., Mackintosh, A. \& Cook, G. 2011. Can in-situ cosmogenic ${ }^{14} \mathrm{C}$ be used to assess the influence of clast recycling on exposure dating of ice retreat in Antarctica? Quaternary Geochronology, 6, 289-294.

Xu, S., Dougans, A.B., Freeman, S.P.H.T., Schnabel, C. \& Wilcken, K.M. 2010. Improved ${ }^{10} \mathrm{Be}$ and ${ }^{26} \mathrm{Al}-\mathrm{AMS}$ with a $5 \mathrm{MV}$ spectrometer. Nuclear Instruments and Methods in Physics Research Section, B268, 736-738. 